\title{
LAPLACE: A mission to Europa and the Jupiter System for ESA's Cosmic Vision Programme
}

Michel Blanc • Yann Alibert • Nicolas André • Sushil Atreya • Reta Beebe • Willy Benz • Scott J. Bolton • Angioletta Coradini • Athena Coustenis • Véronique Dehant • Michele Dougherty · Pierre Drossart • Masaki Fujimoto • Olivier Grasset • Leonid Gurvits • Paul Hartogh • Hauke Hussmann • Yasumasa Kasaba • Margaret Kivelson • Krishan Khurana • Norbert Krupp • Philippe Louarn • Jonathan Lunine • Melissa McGrath • David Mimoun • Olivier Mousis • Juergen Oberst • Tatsuaki Okada • Robert Pappalardo • Olga Prieto-Ballesteros • Daniel Prieur • Pascal Regnier • Maarten Roos-Serote • Sho Sasaki · Gerald Schubert • Christophe Sotin · Tom Spilker • Yukihiro Takahashi · Takeshi Takashima · Federico Tosi • Diego Turrini • Tim Van Hoolst • Lev Zelenyi

Received: 26 February 2008 / Accepted: 6 October 2008 / Published online: 21 January 2009

(C) The Author(s) 2009. This article is published with open access at Springerlink.com

Team members: full list available at http:/jupiter-europa.cesr.fr/.

Full list of LAPLACE proposal members at http:/laplace.cesr.fr.

M. Blanc $(\varangle) \cdot$ P. Louarn

CESR (UPS/CNRS), 9 avenue du Colonel Roche, 31028, Toulouse Cedex 4, France e-mail: michel.blanc@cesr.fr

M. Blanc

Ecole Polytechnique, Palaiseau, France

Y. Alibert · W. Benz

Physikalisches Institut, University of Bern, Sidlerstrasse 5,

3012, Bern, Switzerland

Y. Alibert · O. Mousis

Observatoire de Besançon, Institut UTINAM, 41 bis Avenue de l'Observatoire,

BP 1615, 25 010, Besançon Cedex, France

N. André

Research and Scientific Support Department, European Space Agency, 2200 AG, Noordwijk, The Netherlands

\section{S. Atreya}

Department of Atmospheric, Oceanic and Space Sciences,

University of Michigan, Space Research Building, Ann Arbor, MI 48109-2143, USA

R. Beebe

New Mexico State University, Las Cruces, NM, USA 
Abstract The exploration of the Jovian System and its fascinating satellite Europa is one of the priorities presented in ESA's "Cosmic Vision" strategic document. The Jovian System indeed displays many facets. It is a small planetary system in its own right, built-up out of the mixture of gas and icy material that was present in the external region of the solar nebula. Through a complex history of accretion, internal differentiation and dynamic interaction, a very unique satellite system formed, in which three of the four Galilean satellites are locked in the so-called Laplace resonance. The energy and angular momentum they exchange among themselves and with Jupiter contribute to various degrees to the internal heating sources of the satellites. Unique among these satellites, Europa is believed to shelter an ocean between its geodynamically active icy crust and its silicate mantle, one where the main conditions for habitability may be fulfilled. For this very reason, Europa is one of the best candidates for the search for life in our Solar System. So, is Europa really habitable, representing a "habitable zone" in the Jupiter system? To answer

\section{S. J. Bolton}

SwRI, San Antonio, TX, USA

A. Coradini $\cdot$ F. Tosi $\cdot$ D. Turrini

INAF-Istituto di Fisica dello Spazio Interplanetario,

Via Fosso del Cavaliere 100, 00133, Roma, Italy

A. Coustenis $\cdot$ P. Drossart

Observatoire de Paris, LESIA, 5 place Jules Janssen, 92 195, Meudon Cedex, France

V. Dehant · T. Van Hoolst

Royal Observatory of Belgium, Brussels, Belgium

M. Dougherty

Space and Atmospheric Physics, Imperial College, London, SW7 2AZ, UK

M. Fujimoto $\cdot$ T. Okada $\cdot$ T. Takashima

Japan Aerospace Exploration Agency (JAXA), 3-1-1 Yoshinodai, Sagamihara,

Kanagawa, 229-8510, Japan

O. Grasset

Laboratoire de Planetologie, Université de Nantes, BP 92205, 44322, Nantes Cedex 03, France

L. Gurvits

JIVE, Dwingeloo, The Netherlands

P. Hartogh $\cdot$ H. Hussmann

DLR Institute of Planetary Research, Rutherfordstr. 2, 12489, Berlin, Germany

Y. Kasaba

Department of Geophysics, Tohoku University, Aoba, Sendai, Miyagi, 980-8578, Japan

M. Kivelson · K. Khurana

University of California, Los Angeles, CA, USA

N. Krupp

Max-Planck-Institut für Sonnensystemforschung, Max-Planck-Straße 2,

37191, Katlenburg-Lindau, Germany 
this specific question, we need a dedicated mission to Europa. But to understand in a more generic way the habitability conditions around giant planets, we need to go beyond Europa itself and address two more general questions at the scale of the Jupiter system: to what extent is its possible habitability related to the initial conditions and formation scenario of the Jovian satellites? To what extent is it due to the way the Jupiter system works? ESA's Cosmic Vision programme offers an ideal and timely framework to address these three key questions. Building on the in-depth reconnaissance of the Jupiter System by Galileo (and the Voyager, Ulysses, Cassini and New Horizons fly-by's) and on the anticipated accomplishments of NASA's JUNO mission, it is now time to design and fly a new mission which will focus on these three major questions. LAPLACE, as we propose to call it, will deploy in the Jovian system a triad of orbiting platforms to perform coordinated observations of its main components: Europa, our priority target, the Jovian satellites, Jupiter's magnetosphere and its atmosphere and interior. LAPLACE will consolidate Europe's role and visibility in the exploration of the Solar System and will foster the development of technologies for the exploration of deep space in Europe. Its multi-platform and multi-target architecture, combined with its broadly multidisciplinary scientific

J. Lunine

University of Arizona, Tucson, AZ, USA

M. McGrath

NASA/Marshall Spaceflight Center, Huntsville, AL, USA

D. Mimoun

Université de Toulouse, ISAE-SUPAERO, 10 avenue Edouard Belin, BP 54032, 31055, Toulouse Cedex 4, France

J. Oberst

DLR, Berlin, Germany

R. Pappalardo · T. Spilker

NASA/JPL, Pasadena, CA, USA

O. Prieto-Ballesteros

Laboratorio de Geologia Planetaria, Centro de Astrobiologia-INTA-CSIC,

Ctra. Ajalvir km.4, Torrejon de Ardoz, Madrid, Spain

D. Prieur

Université de Bretagne Occidentale, Brest, France

P. Regnier

EADS/ASTRIUM, Toulouse, France

M. Roos-Serote $\cdot$ S. Sasaki

National Astronomical Observatory, Mizusawa, Japan

G. Schubert

Department of Earth and Space Sciences, Institute of Geophysics and Planetary Physics,

University of California, Los Angeles, CA 90095-1567, USA

C. Sotin

Jet Propulsion Laboratory, 4800 Oak Grove Dr, Pasadena, CA 91109, USA 
dimension, will provide an outstanding opportunity to build a broad international collaboration with all interested nations and space agencies.

Keywords Jupiter • Europa • Jovian system • ESA's Cosmic Vision • Mission

\section{Introduction: a Cosmic Vision of the Jupiter system}

Galileo Galilei's January 1610 observations of the four main satellites of Jupiter represent in a sense the first time a "planetary system" was ever observed from outside. Today, while we prepare the celebration of the 400th anniversary of this break-though in our understanding of the Solar System, the place of the Jupiter System in planetary science and discovery is even more central:

- The most recent simulations of the dynamical evolution of the early Solar System show that Jupiter has played a key role in the formation and early evolution of its sister planets and of the populations of small bodies and has controlled the amount of water delivered to terrestrial planets, thus influencing the habitability and emergence of life on our own planet.

- With the discovery of over 200 extrasolar planets since 1995, Jupiter appears as a "template", available in our own Solar System, for the numerous gas giants found around other stars. Progress in our understanding of how Jupiter and its satellite system were formed and migrated, and of how Jupiter works to-day, can therefore be translated into an improved basis for the planning and later understanding of observations of extrasolar planets.

- Finally, the naïve idea that the Jupiter system is indeed a "mini-solar system" has been only reinforced by the Pioneer, Voyager, Ulysses, Cassini and recently New Horizons fly-bys of the system, and by Galileo's extended orbital tour. The Voyager fly-by of its moons first revealed the unique aspects of its satellite Europa as a potential "ocean moon", and opened the possibility for the existence of a "habitable zone" in the Jupiter system. Moreover, simulation studies strongly suggest that the formation scenario of the Jupiter system displays many similarities with the formation of the solar system as a whole, involving the dynamical evolution and condensation of a Jovian sub-nebula.

- Thus it is no surprise that the Jupiter System plays such a prominent role in the Cosmic Vision Plan proposed by the European Agency to build its ambitious scientific programme over the period 2015-2025. Three key questions about the Jupiter System and its habitability (Fig. 1) strongly resonate with the major scientific questions identified by the Cosmic Vision Plan.

Y. Takahashi

Tohoku University, Aoba, Sendai, Miyagi, 980-8578, Japan

L. Zelenyi

Space Research Institute (IKI), Moscow, Russia

Springer 
Fig. 1 The three main scientific questions of the mission and their interrelations

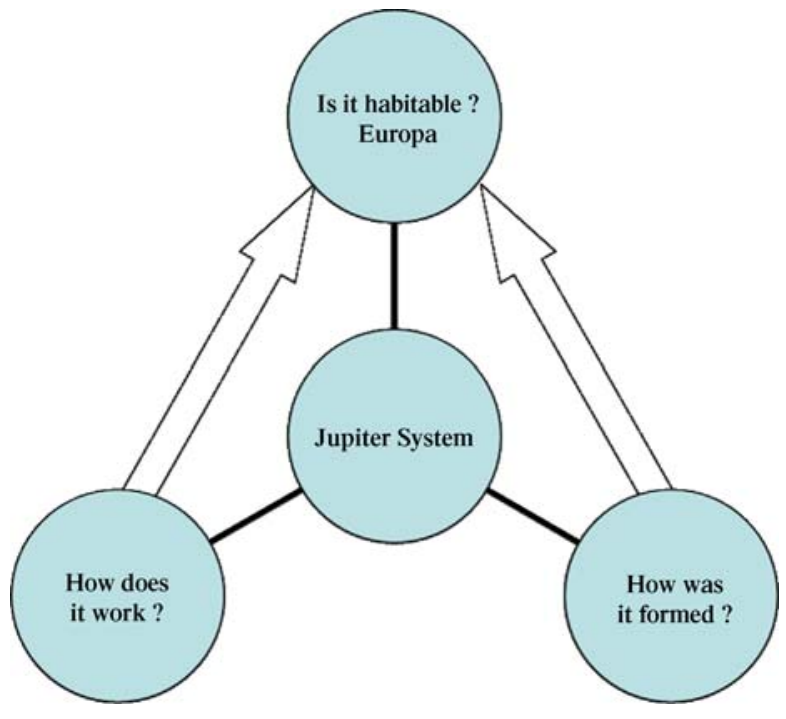

They are the Science Goals of the LAPLACE mission:

- What have been the conditions for the formation of the Jupiter system? How do they relate to the formation scenario of the solar system and other planetary systems? How do they contribute to the possible emergence of life? (Cosmic Vision theme 1).

- How does the Jupiter system work? How well do we understand it at the "system level"? (Cosmic Vision Theme 2). How does the system contribute to the conditions for habitability?

- Is Europa habitable

Does it represent the "habitable zone" of the Jupiter system? Does Europa actually harbour life? (Cosmic Vision Theme 1).

\section{Scientific objectives}

To assess Europa's habitability, our priority science goal, we first need to understand how the Jupiter system was formed (science goal 1), and what coupling processes link its many objects (science goal 2). We will then focus on our in-depth exploration of Europa.

\subsection{How did the Jupiter system form?}

\subsubsection{Formation scenarios for the Jupiter system}

The history of the Jovian system can be divided into three main phases: the formation of Jupiter, the formation of its satellite system and its secular evolution to its present day state. The first phase (first line in Fig. 2), the formation of Jupiter, took place during the nebular epoch of Solar System history, when the gaseous component was 


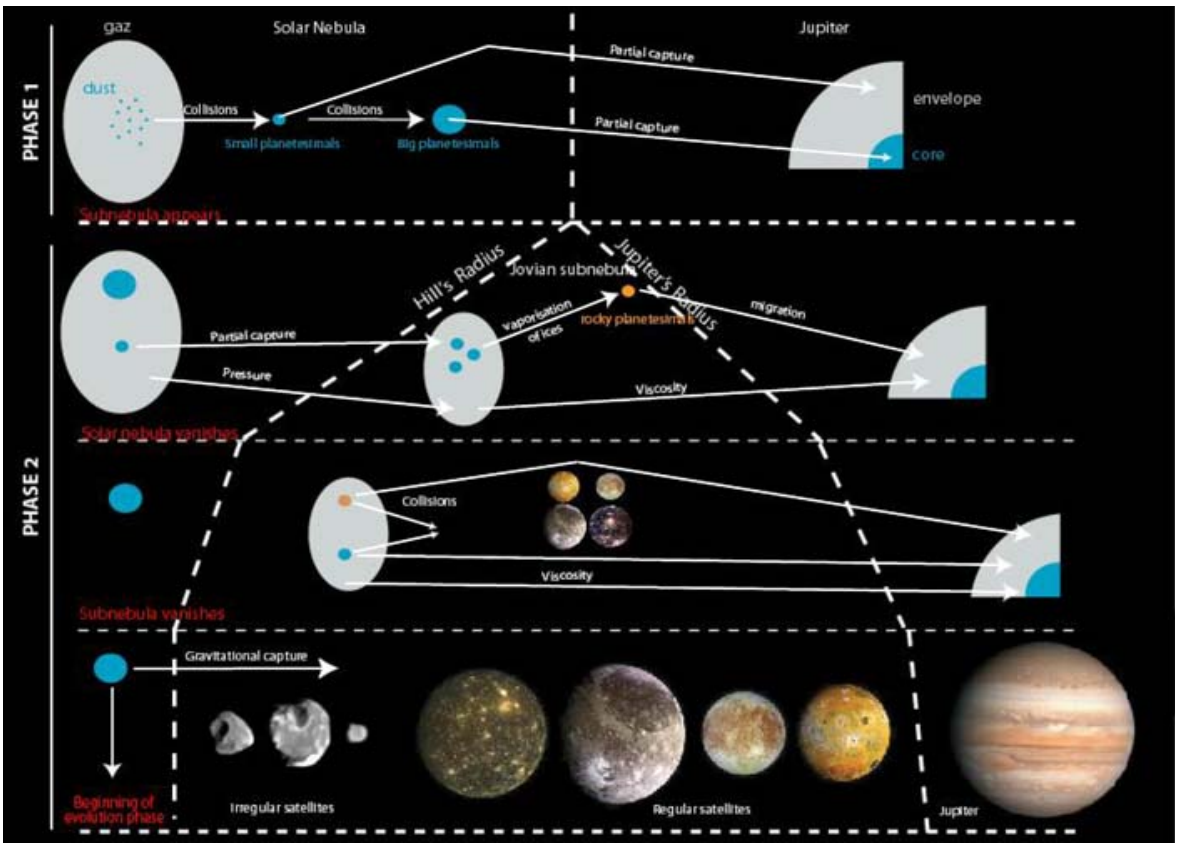

Fig. 2 A scenario of Jupiter system formation. First phase: Jupiter forms, its Hill and outer radii coincide, and no subnebula can exist (first line). Second phase: formation of the satellite system. A subnebula appears, fed in its infancy by gas and gas-coupled solids originating from the solar. Planetesimals, some of them having lost their volatile due to temperature and pressure conditions prevailing in the subnebula, grow to form satellites (second and third lines). When the subnebula disappears (fourth line), the irregular satellites are captured

still present and the solid component was slowly giving birth to planetesimals by means of accumulation processes. Two competing models are generally proposed to explain the formation of giant planets.

In the first approach, the nucleated instability model [2, 50, 70], one assumes that planetary cores form by accumulation of planetesimals, in a way similar to the one generally accepted for the formation of terrestrial planets. When a critical mass is reached, the cores start to capture ever increasing quantities of nebular gas, leading to a phase of gas accretion where the planets grow to their final masses [50]. This scenario has been shown to be compatible with our present day very limited knowledge of Jupiter's internal structure and atmospheric composition. The second scenario $[8,52]$ assumes that giant planets are formed by gravitational instabilities in the massive proto-Solar Nebula [7], leading to the rapid formation of density enhancements-or clumps-with solar chemical compositions and masses probably larger than the present ones of the giant planets. Key issues with this model are how to justify the a posteriori formation of a core and the large amount of heavy elements suspected, but not yet fully determined; the exception being the supersolar $\mathrm{C}, \mathrm{S}, \mathrm{N}$, $\mathrm{Ar}, \mathrm{Kr}$ and $\mathrm{Xe}$ abundances (relative to $\mathrm{H}$ ) in Jupiter's troposphere [5] and only $\mathrm{C} / \mathrm{H}$ at Saturn [25]. See also [32].

The second phase (second to fourth lines in Fig. 2) begins when Jupiter's region of gravitational influence (quantified by its Hill's radius) enlarges and exceeds its 
outer radius. This results from the accretion of a massive gas envelope, and leads to the generation of a Jovian subnebula, a structure similar to the Solar Nebula, which had the ability of capturing gas and small planetesimals from it [3, 10, 11, 62]. The captured planetesimals then can grow further in mass due to mutual collisions and build up the regular Jovian satellites (Fig. 2, second and third lines): the content of ices can vary from satellite to satellite, since part of the original planetesimals could have lost their volatile species (in particular water ice) due to the temperature and pressure condition prevailing in the Jovian subnebula [63]. At the end of this process, all of the subnebular gas and the majority of its small scale solids would be accreted into Jupiter. The formation of the Jovian regular satellite system ended at an epoch between the subnebular phase and the Late Heavy Bombardment, an event which has recently been suggested to have been triggered by a chaotic phase of orbital rearrangement of the outer Solar System [60]. Depending on the capture mechanism, the presence of subnebular gas or the dynamical rearrangement could have supplied the conditions necessary for the gravitational capture of the irregular satellites of the giant planets.

Finally, the completely formed Jovian system would have started its evolution phase towards its present day state, as a consequence of impacts and the various coupling processes acting inside the system.

\subsubsection{LAPLACE contribution in unveiling the origin of the Jovian system}

Understanding the formation of Jupiter and its satellite system therefore requires that we look at the traces of the first and second phases just described above. The strategies devised for the LAPLACE mission are presented in the three points below, complemented by composition measurements of regular satellites (see Section 3.2).

Probing Jupiter's interior In order to determine Jupiter's internal structure, two approaches are available. The first one, which will be used by the NASA's 2011 JUNO mission, relies on the determination of the planet's gravitational moments. Since their interpretation in terms of density structure and of the presence or absence of a core is not unique and strongly depends on the correct knowledge of $\mathrm{H}$ and $\mathrm{He}$ equations of state, uncertainties in the equations of state will affect the achievable resolution.

An alternative way to obtain information on Jupiter's interior is the seismology approach, namely the study of the oscillations of the planet. Observations aimed at detecting oscillation modes of Jupiter have shown promising results [77], but so far they have been limited by instrumental and windowing effects and by the effect of Earth's atmosphere [32]. Combining this method with the gravitational moment data which JUNO will provide has the potential of determining the whole internal density profile of Jupiter, thus giving the mass of its internal core and the amount of heavy elements. It can also constrain the level of homogeneity of Jupiter's envelope, key information to interpret Galileo measurements of volatile species in a global context.

Looking for the origin of the irregular satellites The study of the irregular satellites will allow testing of the capture hypothesis, the reality of the dynamical families found from Earth and defining their physical nature without having to rely only on the information-poor colour indexes and the limited spectral range accessible with ground-based observations. LAPLACE will perform at least one close fly-by 
of one of the major irregular satellites and will remotely observe as many of them as possible, with special emphasis on possible family members of the close fly-by target. It will perform high-resolution imaging and visual-near infrared spectroscopy of the satellite surfaces and dust collection and analysis (dynamics and composition) in their orbital region and during the fly-bys. Observing the Jovian irregular satellites also offers the possibility to look back to the early phases of the evolution of the Solar system and sample the conditions that existed in the primordial planetesimal disk.

Reading the history of the Galilean satellites in their surface morphology The study of the cratering history of the Galilean satellites will provide information to date the Jovian system and the epoch of the Late Heavy Bombardment and the possibly related chaotic orbital rearrangement of the giant planets. High resolution imaging and visual/near infrared spectroscopy of a few selected site, coupled with coverage of the satellites surfaces at a medium resolution (better than Galileo) will provide us with new insight into the history of the Jovian and, more generally, the Solar System.

\subsection{How does the Jupiter system work?}

\subsubsection{A diversity of objects and coupling processes}

The Jupiter system comprises a broad diversity of objects, including Jupiter itself, more than 60 outer irregular small satellites (1 to $100 \mathrm{~km}$ class objects), the four large Galilean Satellites, Io, Europa, Ganymede, and Callisto (1,000 km class objects), the four inner satellites Metis, Adrastea, Amalthea and Thebe (10-100 km class objects) and the Jovian ring system, located in the inner region. All these objects experience gravitational interactions among themselves, and electrodynamic interactions of variable geometries and strengths with Jupiter's magnetospheric particles and fields. The magnetosphere itself is strongly coupled to Jupiter's upper atmosphere and interior by electrodynamic interactions. The complex interactions between these components (see Figs. 3, 5 and 6) redistribute momentum and energy among them

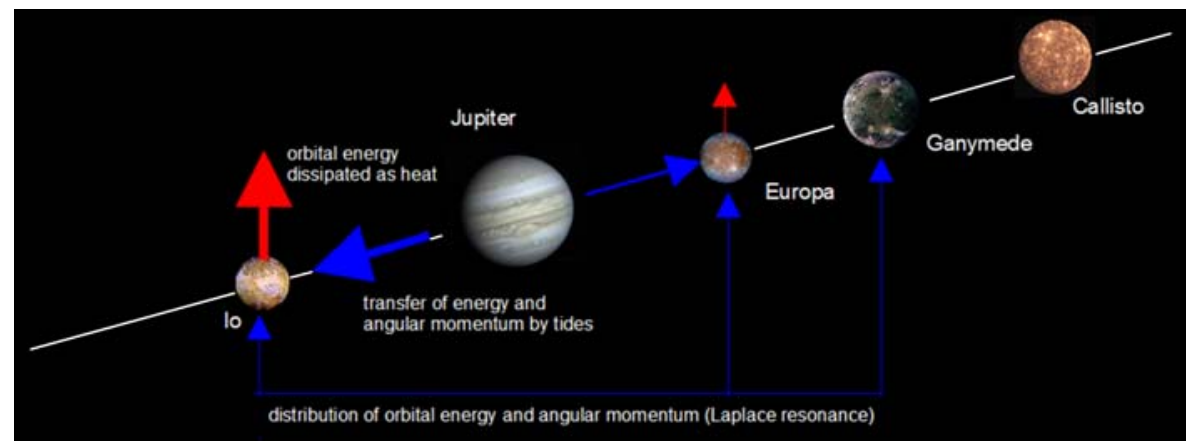

Fig. 3 The rotational energy of Jupiter is a huge reservoir of energy for the three inner Galilean satellites. Orbital energy gained by Io due to tidal torques exerted by Jupiter is distributed among Io, Europa, and Ganymede, due to the Laplace resonance. The resonance is therefore essential for ongoing tidal heating inside Europa, and may allow for the existence of an ocean inside Europa over billions of years 
and drive the magnetospheric engine which generates Jupiter's radiation belts. Finally, redistribution of momentum and energy among different layers of Jupiter's atmosphere is governed by hydrodynamic and radiative processes (for an overview on the Jupiter system, see [6]).

\subsubsection{Gravitational coupling, structure and composition of Galilean Satellites}

The astonishing diversity of features which shape the surfaces, interiors and dynamics of the Galilean Satellites still challenges our understanding: the most intense volcanic and geologic activity in the solar system is found on Io (e.g., [26]) subsurface oceans are likely to exist within Europa, Ganymede and Callisto (e.g., [65, 81]) the smallest solar system magnetosphere is found at Ganymede [40, 41], comparable only to Mercury. The diversity of geologic features on Europa [28] and Ganymede [66], or the incomplete differentiation of Callisto [78] and the completely different evolutionary paths of all four Galilean moons, are all still waiting for a satisfactory explanation. Still, all this diversity must result from the same mechanisms which shaped and still drive the evolution of their interiors and surfaces. Among them, the Laplace resonance between Io, Europe and Ganymede plays a particularly important role in dragging energy from Jupiter to maintain tidal heating at these satellites, and to drive their internal dynamics and modify their surfaces [85]. Interactions with the exosphere and magnetosphere also shape their surfaces via space weathering [36]. Understanding and untangling these driving factors is an essential prerequisite to any attempt at detecting biosignatures at the surface of Europa and possibly of its sister moons.

The Laplace resonance and tidal heating Stable resonances by which orbital periods are kept in a fixed ratio play an essential role in the Jupiter system. Unique in the solar system is the three-body resonance-the so-called Laplace resonance-by which the orbital periods of Io, Europa, and Ganymede are kept in a ratio of 1:2:4.

The resonance maintains the orbital eccentricities of the moons and therefore enables tidal interactions to operate on geological timescales [29, 69, 85]. The vigorous volcanism of Io [26, 55], the innermost Galilean satellite, is the most obvious consequence of internal heating due to tidal friction.

As illustrated in Fig. 3, gravitational coupling redistributes rotational energy and angular momentum between the three moons and Jupiter. The resulting tidal heating significantly contributes to the internal heat sources of Io and of Europa. Europa's tidal deformation and Io's intrinsic heat flow derived from mapping surface temperatures will yield constraints for the amount of energy dissipated inside Io and Europa. The thermal activity of the moons will be inferred from searching for temperature anomalies at the surfaces. Additionally, astrometric determination of the satellites' positions will precisely characterize the orbital state of the system. By studying the Laplace resonance and tidal heating in the Jupiter system, constraints for the habitability of Europa over geologic timescales can be inferred.

Internal structure of the Galilean satellites Models of the internal structure of the Galilean satellites are mainly based on the determination of the mean density and the moment of inertia about the rotational axis by the Galileo mission (e.g., [78]). However, hydrostatic equilibrium assumed in such models has been directly 
confirmed only at Io, where polar and equatorial flybys could be analyzed independently. Whereas Io, Europa and Ganymede are strongly differentiated into an outer ice layer, a rock mantle and an iron rich core, these phases are only partly separated in Callisto although its size and mean density is very similar to Ganymede's. How these different states came to be is still an open question. The low-degree static gravity field of the Galilean satellites has been determined with a precision of a few percent from flybys by the Galileo mission [78]. A Europa orbiter as part of the LAPLACE mission will obtain detailed information on the gravity field of Europa allowing for a great improvement of internal structure models. Moreover, LAPLACE will measure the topography of Europa, which in case of the absence of gravity anomalies can constrain ice shell thickness variations. The degree of differentiation of Europa will be inferred from determining the moon's obliquity, constraining the moment of inertia independently. For the other satellites an order of magnitude improvement of the gravity field is expected by using radio and VLBI tracking from the Earth during the flybys, coupled with altimeter, camera and astrometric (both ground-based and space) data. Deviations from hydrostatic equilibrium and local mass anomalies could be detected and characterized.

One of the most intriguing features with important astrobiological implications is the possible presence of subsurface oceans inside the icy moons of Jupiter (e.g., [81]). Evidence for liquid water layers inside Europa, Ganymede and Callisto has been obtained by Galileo mainly by the detection of induced magnetic fields generated close to the surface at all three satellites (e.g., [46]). In the case of Europa observed surface features related to tectonics and cryo-volcanic processes (Fig. 8) provide additional evidence [65]. Possible structures of the $\mathrm{H}_{2} \mathrm{O}$ shells of Europa, Ganymede and Callisto are shown in Fig. 4.

To further constrain these models and to provide unambiguous evidence for these oceans, in particular at Europa, is a key goal of LAPLACE. Whereas the ocean in Ganymede and Callisto is expected to be enclosed by thick $(\sim 100 \mathrm{~km})$ ice layers, the ocean of Europa can be close to the surface $(\sim 50$-few $\mathrm{km})$ and in contact with silicate rock.

LAPLACE will determine the presence of oceans by measuring the tidal response in addition to the detection and characterization of the induced magnetic fields of the satellites. The large tides predicted for Europa, with estimated amplitudes of about $30 \mathrm{~m}$ if a subsurface ocean exists and only a few $\mathrm{m}$ without an ocean [59], will be measured from the gravitational perturbations on the spacecraft and from altimeter measurements with a precision of about $1 \%$. In combination with determining the libration amplitudes (order of $100 \mathrm{~m}$ at Europa's equator) this will constrain the thickness of the icy shell [84]. Independent from tidal interaction LAPLACE will measure the electromagnetic response, i.e. the induced magnetic fields, of the ocean at multiple frequencies at Europa. This will constrain the thickness of the ice shell, the thickness of the ocean and its electrical conductivity. Similar measurements during flybys will allow for the detection and characterization of subsurface oceans at Ganymede and Callisto with reduced precision as compared to Europa, depending on the number and geometry of the flybys.

Galileo has found a self generated magnetic field of Ganymede [40, 41], a unique feature among solar system satellites. To better understand the magnetic field generation in the iron core and to constrain interior structure and evolution models of Ganymede, a detailed characterization of the field structure will be obtained. 
Fig. 4 Possible locations of liquid layers in the icy moons of Jupiter are plotted here as a function of depth: 1 completely frozen; 2 three-layered structures impeding any contact between the liquid layer and the silicate floor; 3 thick upper icy layer $(>10 \mathrm{~km})$ and a thick ocean; 4 very thin upper icy layer (3-4 km). Structures 3-4 are the most probable for Europa (indicated in black in the lower part). The larger moons Ganymede and Callisto are located in the left region (1 or 2) where internal pressures are sufficient to allow for the formation of high-pressure ice-phases. Oceans in Ganymede and Callisto-if they exist-should be enclosed between thick ice layers

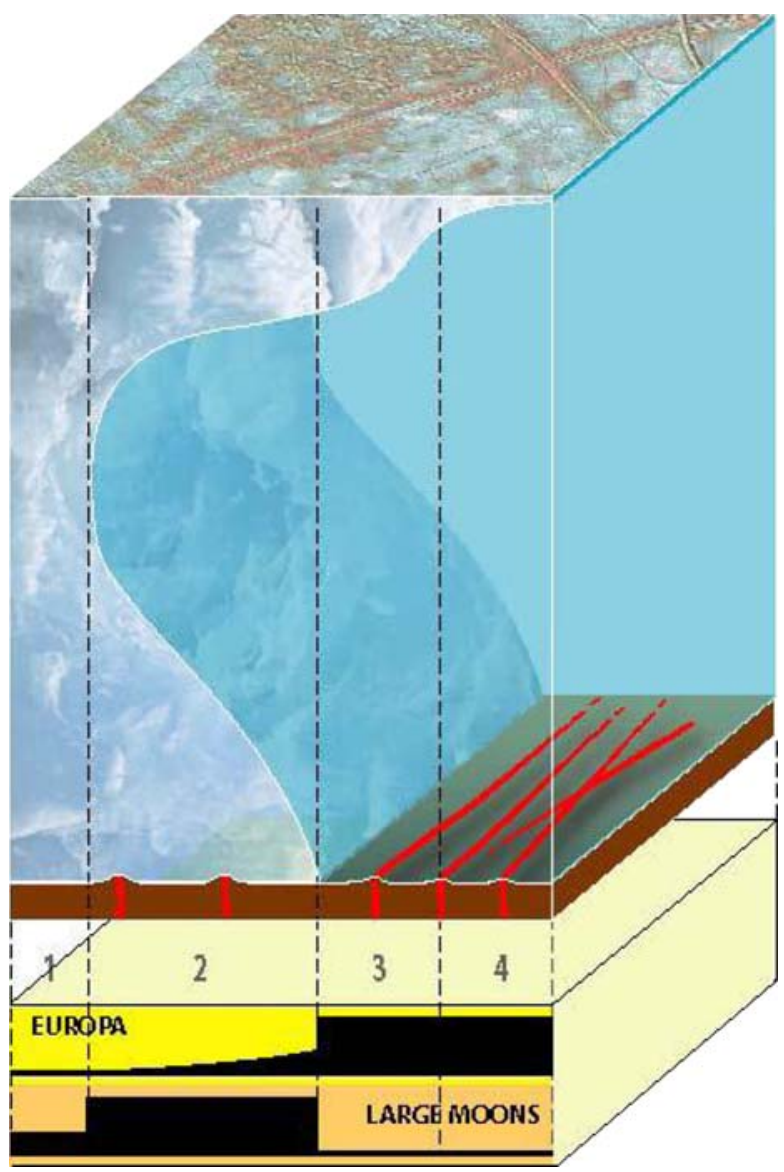

Physical characteristics, composition and geology of the surfaces of the Galilean Satellites The surfaces of satellites contain valuable information of the moons' histories. Surface features were formed by external sources including impacts and particle bombardment and by geologic activity caused by internal dynamics. The Galilean Satellites offer a perfect illustration of these two processes: the surface of Io is almost completely formed by endogenic activity [26, 55], whereas Callisto's surface was almost entirely altered externally early in solar system history [57]. At Europa and Ganymede different phases of endogenic activity have formed the complex icy surfaces of these bodies [28, 65, 66]. The inner satellites are exposed to stronger radiation in the inner part of the Jovian magnetosphere which leaves its traces at the surfaces and exospheres of the satellites [36]. LAPLACE will study these surface processes by a diversity of approaches:

- Geologic mapping, including significant improvement of global coverage

- Laser altimetry or stereo imaging to derive topographic maps

- Searching for geologic activity from limb scans, thermal anomalies, and image comparison 
- Monitoring of Io's volcanic and thermal activity on day- and nightside

- Determination of global and regional surface ages from the cratering record

- Characterization of the non-ice components and search for organic compounds

- Determination of chemical, elemental and isotopical composition by dust collection and analysis.

- Mapping of surface alterations due to the radiation environment

Our goal is to improve the global coverage of surface imaging and spectroscopy, compared to Galileo, for all Galilean Satellites (50\% of surfaces with 200 and $500 \mathrm{~m} / \mathrm{pxl}$ for total surfaces in the visible and near infrared ranging from 350$5,000 \mathrm{~nm}$ ). High resolution images at local regions at $<10 \mathrm{~m} / \mathrm{pxl}$ will be obtained from close flybys.

\subsubsection{Electrodynamic coupling and the jovian magnetospheric engine}

Just like gravitational coupling, electrodynamic interactions also operate at a global scale within the Jupiter system. Magnetospheric charged particles, mainly produced by the ionization of Io's neutral torus, populate the whole Jovian magnetosphere and form an extended magnetodisk near the magnetic equator [6, 22]. This magnetodisk interacts with each satellite in a variety of modes, depending on the satellite's internal plasma sources, on the properties of its exosphere and surface, and on its degree of magnetization. It is also coupled to Jupiter's rotation via a system of electric currents which flow along magnetic field lines and into Jupiter's upper atmosphere, where they generate the main auroral oval $[27,72]$. The complex interplay between plasma generation at the satellites, satellite-magnetosphere interactions and Jupitermagnetosphere interactions, produces the Jovian radiation belts [23], the harshest radiation environment in the Solar System, which directly affects habitability on the surfaces of the jovian moons (Fig. 5).

The jovian magnetosphere-basically a giant magnetized environment, driven by the fast rotation of its central spinning object and populated by ions coming mainly its moons [38] - is thus the most accessible environment for a direct investigation of general astrophysical processes regarding: (i) the dynamics of magnetodisks, with different mechanisms of angular momentum exchange and dissipation of rotational energy ("fast rotator" theme), (ii) the electro-dynamical coupling between a central body and its satellites ("binary system" theme) and, (iii) the global and continuous acceleration of particles ("giant acceleration" theme).

The fast magnetic rotator The fast jovian rotation, combined with the continuous creation of ion populations due to Io's volcanism, leads to the formation of a magneto-disc [39]. Its structure varies with distance from Jupiter. Starting at Io's orbit as a torus of neutrals and ionized particles in rigid co-rotation, the torus progressively thins and becomes a disc-like system of limited vertical extension. It increasingly departs from rigid co-rotation with the distance to Jupiter, with magnetic effects as important as the thermal and rotational ones. At 50-60 Rj from Jupiter, in the antisolar direction, the disc transforms into a magnetotail with a strong 'planetary' wind.

This disk is driven in rotation by an electro-dynamical coupling with Jupiter materialized by a system of currents circulating along the magnetic field lines and closing at the ionosphere. This current system transfers angular momentum from the outer layers of Jupiter atmosphere to the disc. As this coupling is not perfect, 


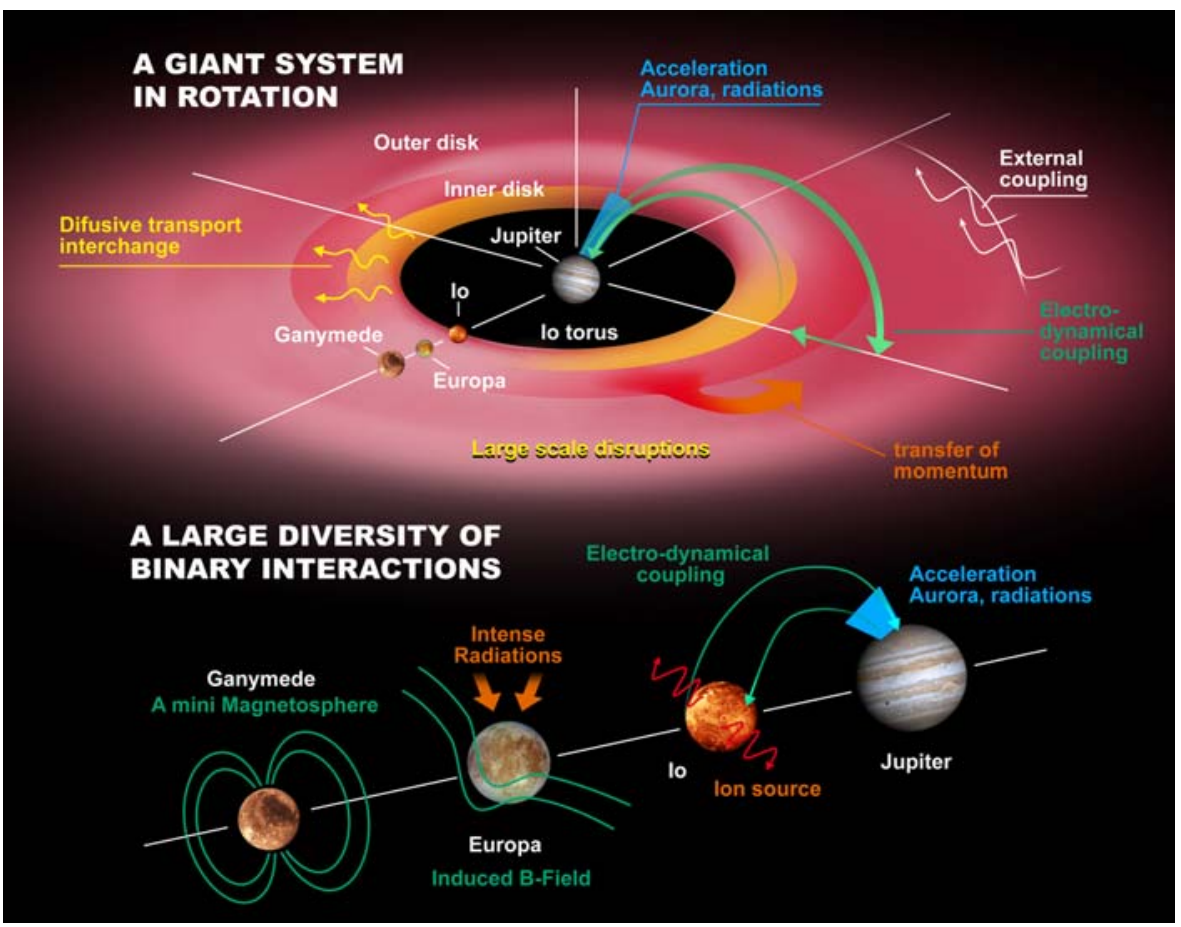

Fig. 5 Electrodynamic interactions play a variety of roles in the Jupiter system: generation of plasma at the Io torus, magnetosphere/satellites interactions, dynamics of a giant plasma disc coupled to Jupiter's rotation by the auroral current system, generation of Jupiter's intense radiation belts

it leads to particle acceleration, resulting in auroras, energy deposition in the upper layers of the atmosphere and powerful radio emissions. These effects are particularly important where co-rotation breaks down, at 15-25 $\mathrm{Rj}$ [34].

The structure of the disc and its coupling with Jupiter also strongly vary with local time. Establishing the 3D structure of this disk, and its variability, is an important goal of a new exploration of Jupiter, crucial for further analysis of all angular momentum transfer processes in magnetized, ionized media.

Indeed, the disk - this 3D structure-is not a stationary system, it is highly dynamic. Galileo observations have revealed that it is periodically reconfigured over global scales, with sequences of loading-unloading of the disk by new plasma and injections of energetic populations both in the inner magnetosphere and the outer disk. Various processes contribute to the radial transport, from the Io torus to the external magnetosphere and, at the end, the interplanetary medium: microscopic diffusion, meso-scale interchanges, global sporadic disruptions of the disk and magnetic reconnection. All these processes trigger variations in the transport rate of the iogenic plasma and its radial re-distribution [4, 42, 48, 71]. The chain of processes involved in the exchanges of angular momentum and the dissipation of rotational energy, from the global dissipation of the rotational energy to the microscopic mechanisms responsible for non-ideality is unknown. Determining their 
spatial/temporal scales, quantifying their relative efficiency and how they vary with the activity of Io is a central objective.

As all objects in the solar system, Jupiter interacts with the solar wind which drives a part of the magnetospheric dynamics and possibly triggers major storms when solar perturbations hit the magnetosphere. To what extent is this huge system, endowed with its own powerful dynamics, affected by and responsive to external perturbation? This question concerning the respective importance of external and internal processes in the regulation of the activity of a magnetic environment can also be best studied at Jupiter.

The magnetized binary system Different 'objects' move in the Jovian environment, each of them interacting with the magnetopheric plasma by a large variety of processes. Moons, with their exospheres, are conductive bodies. As they move trough the Jovian magnetic field, they create a specific current system (the unipolar dynamo). This electro-dynamical coupling is not stationary. It generates Alfvèn wave structures, called 'Alfvèn wings, that couple the jovian ionosphere to the exosphere of the moons. Once again, this coupling is not perfect. Part of the electromagnetic energy is converted into kinetic energy of accelerated particles, with the formation of particular auroral features, including the generation of non thermal radio emissions [15, 33, 40, 41].

Europa, Ganymede and Callisto are in complementary situations [64]. The interaction at Io is the most powerful. It has an extended exosphere, able to fill the environment with matter, and moving in a dense and highly magnetized plasma (the inner Io torus). Ganymede, the unique (to date) magnetized moon, constitutes another remarkable situation. We don't know how this mini-magnetosphere is coupled to Jupiter's huge one. We only know that this interaction is powerful enough to create an intense auroral footpoint at Jupiter. The coupling with Europa is apparently much less powerful, even if it seems able to generate intense waves. By contrast, Callisto is the most quiet.

The parameters that determine the strength of the coupling, the way magnetic fields are distorted and large-scale fluctuations are generated, are ignored, as are the details of the interaction itself. Furthermore, the moons are not passive, more or less conductive bodies. Each moon generates a large level of plasma waves, they locally heat and accelerate the ion populations, enhance the spatial diffusion and lead to a more efficient assimilation of the exospheric ions by the Jovian magnetosphere.

The Io torus is another original system. In some sense, its innermost region presents similarities with an ionosphere, where populations of neutrals interact with ionized ones. However, it is fundamentally different in terms of boundary conditions, spatial scales, geometry and forces that regulate the equilibrium. How does this system evolve when the volcanic activity of Io changes? How are the different ion species mixed, and at what scales? What is the importance of turbulence? How does the torus feed the magneto-disk? Answers to these questions will greatly help to quantify fundamental mixing, heating, energy exchange processes in dilute, multiphase media.

All these topics relate to whether a planet immersed in a strong radiation environment can host complex compounds able to react chemically. They are also of central importance for the interpretation of precise spectral analyses of Europa surface, dedicated to the search for pre-biotic components. 
The giant particle accelerator A further, largely unresolved problem of a fundamental nature regards the Jovian magnetosphere as a tremendously efficient particle accelerator $[47,76]$. There is no question that Io is the primary source of particles in the Jovian environment. The new plasma populations have, however, typical energies of a few 10's eV at best. At what time and spatial scales, and by what mechanisms, does a significant part of them reach $\mathrm{MeV}$ energies and populate the harshest radiating environment of the solar system? The fact that such environments can exist has direct relevance to the notion of 'habitability'. A large and highly variable level of radiation may actually modify or control the emergence of life.

Jupiter is also a 'prototype' of powerful exo-magnetosphere, possibly powerful enough in radio to be detected at stellar distances. One interesting outcome of insitu measurements in this radio sources is to determine the highest level of wave energy reachable inside the radio sources and then, to better estimate their possible detection by a very distant observer.

Critical measurements with LAPLACE The basic magnetospheric measurements concern the characterisation of the different plasma populations (electrons and ions species) thus, the density, velocity and pressure, including their distribution functions. This requires plasma mass spectrometers capable of making measurements at sufficiently high energies (up to a $100 \mathrm{Kev} / \mathrm{q}$ ) and dedicated high energy instruments. The characterisation of electromagnetic field will be done by measuring the static magnetic field, the magnetic and electric components of high frequency fluctuations.

On orbiting spacecrafts, neutrals and dust must be determined, with possibilities of chemical characterisation. Complementary diagnostic measurements in visible, IR, $\mathrm{UV}, \mathrm{X}$ that will be used to monitor the aurora, the energy deposition in Jupiter atmosphere and, the variability of the Io torus are needed. Sequences of simultaneous in-situ measurements and remote sensing observations of 'magnetospheric' targets (aurora, torii) are required.

The determination of scales, temporal and spatial, is fundamental. They define the dynamical processes and guide any theoretical or simulation analysis. LAPLACE, with the possibility of performing simultaneous measurements in at least two points, will permit completely original analysis. The first phase of the orbital insertion, with spacecraft exploring the magnetosphere, at variable inter-spacecraft radial and azimuthal distances, will be fundamental. The main requirement is to broaden as much as possible the parameters of the exploration, in terms of local time, distances from Jupiter and inter-spacecraft distances.

\subsubsection{Radiative and hydrodynamic coupling in the jovian atmosphere}

Even after the GALILEO mission, some of the most important questions concerning the atmosphere of Jupiter remain: How does Jupiter radiate its internal energy to space, and what are the dynamics and coupling in its atmosphere? LAPLACE will address these questions in a very complementing way to JUNO, extending the measurements to low latitude and equatorial atmosphere and focusing on different scientific questions. Three different layers will be sounded to address these questions.

The upper atmosphere This outermost layer is connected to outer space, and its mesospheric, thermospheric and ionospheric structure and dynamics must be fully characterized. Despite three decades of measurements of Jupiter's upper atmosphere 
from ground and space, our knowledge and understanding of the region still remains in its early infancy. LAPLACE will address three science topics which are closely coupled. Firstly, the thermosphere of Jupiter, like those of all gas giants, is much hotter than expected from the solar EUV heating alone [9]. The energy balance on Jupiter and other gas giants is not yet understood. This will continue to remain mysterious even after JUNO observations in 2016. A systematic measurement of waves on Jupiter at low to mid latitudes is required. The results would have implications for all gas giants in our solar system and the extrasolar systems. Secondly, despite limited observations from the ground, observations by Galileo and forthcoming observations by JUNO, the dynamics of the thermosphere at low and mid latitudes will remain poorly known. For example, it is unclear whether the zonal jets observed in the stratosphere are present also in the thermosphere and the importance of wave acceleration and ion drag in thermospheric winds is unclear [56]. Knowledge of the dynamics is therefore crucial in order to understand how energy and material are distributed horizontally and vertically in the upper atmosphere. Thirdly, a major unknown on Jupiter is its ionosphere, not only its structure, but also its relation to the $\mathrm{H}_{2}$ bulge, the nature of the most important ionization processes, the distribution of ions and the nature of energy deposition processes. Only few vertical profiles of Jupiter's ionosphere are known, and each of them differing substantially from the others, and none of them have been reproduced by photochemical models [1]. LAPLACE will perform a systematic exploration of total electron densities via radio science, and infrared observations at low latitudes will trace $\mathrm{H}_{3}^{+}$, a key molecule in Jupiter's ionosphere [24]. LAPLACE will shed light on the nature of coupling between the thermosphere, ionosphere and magnetosphere of Jupiter at low latitudes, which is essential to understand the processes that drive the entire coupling between the planet and outer space as well as the outer atmosphere and deeper interior of Jupiter. This also has key implications for our understanding of non-thermal atmospheric loss, and thereby of the atmosphere evolution.

The stratosphere The stratosphere couples the deeper layers of the troposphere to the upper atmosphere. Its structure, circulation and composition are still poorly determined. Direct estimation of winds is not possible in the absence of discrete clouds. However, knowledge of the stratospheric circulation is vital in understanding the transport of hazes and minor species. Temperature fields are known from Voyager/Cassini and ground observations, but temporal variations are organized along many different time scales, the longer known being the quasi-quadriennal oscillation, observed from the ground. Wind measurements in the stratosphere are known only from thermal wind equation retrieval, which unfortunately excludes the equatorial regions where important phenomena take place, and from the dispersion of dust and chemical elements since the Shoemaker-Levy 9 collision in 1994 [61]. Missing elements are, to date, clues on the meridional and equatorial circulations and the vertical structure of the winds. Moreover, the question of the origin of stratospheric water is still open, and has implication on the origin of water in the Solar System: latitudinal variations of $\mathrm{H}_{2} \mathrm{O}$ would provide important clues on this origin. Finally, the remnants of the Shoemaker-Levy 9 collision (CS, HCN) are still observed 13 years later and used as tracers of stratospheric mixing. LAPLACE will follow up these observations, in relation with ground-based or space-borne observations (ALMA, Herschel). 
The troposphere The troposphere will be the deepest level sounded by LAPLACE: the tropospheric meteorology in a global sense is poorly understood. In particular, the exact origin of the global circulation of Jupiter, the structure of the band system, its relation to differential rotation and the connection of this meteorological system with deep and outer layers are unknown. One way of progressing in this field is to constrain models by direct measurements of the quantities involved in meteorological equations, such as velocities, thermodynamics quantities and the "potential vorticity", which is conserved in non-dissipative flows like a passive tracer, and which is directly calculated in the models [73]. This latter quantity can be deduced from observations of the wind field, together with temperature profiles of the atmosphere. Moreover, long term monitoring of Jupiter at medium scales $(300 \mathrm{~km})$ with potential vorticity retrieval at different time scales would constrain the evolution of waves, of atmospheric structure and of winds from the models. The distribution of lightning storms on Jupiter, detected by Galileo with a non-uniform density, is still mysterious. The importance of local convection as a source term for global circulation remains to be proven at a global scale. These convective cells can also be a source of strong gravity waves propagating up to the thermosphere, and contributing to upper atmosphere heating. Finally, internal waves already described in Section 3.1 connect the troposphere to the interior of Jupiter and complete the picture that LAPLACE could give of the Jupiter atmosphere from the uppermost layers to the deep interior (Fig. 6).

\subsection{Looking into Europa to assess its habitability}

\subsubsection{How can an icy moon be habitable?}

The potential habitability of Europa rests on the fulfilment of four conditions (Fig. 7): the presence of liquid water, an adequate energy source to sustain the necessary metabolic reactions, a source of chemical elements (C,N,H,O,P,S, etc), which can be used as nutrients for the synthesis of bio-molecules, and relevant pressure and temperature conditions [18, 20, 21, 35, 37, 51, 74, 79].

The fulfilment of the first condition is directly related to the putative existence of an ocean. The existence of this ocean has been inferred from the interpretation of the induced magnetic field measured by the Galileo mission [43, 44] and the interpretation of geological features (Fig. 8) [13, 65].

Europa is not unique among the four Galilean satellites, as subsurface oceans may also exist at Ganymede and Callisto [45, 58, 75]. But according to current models, it represents the only case in which liquid water is in contact with a silicate core. Such conditions are favourable for interactions between the ocean and silicates, particularly if a volcanic, and consequently hydrothermal activity exist; [49, 86, 87]. This would provide a variety of chemicals that could play a role in sustaining putative life forms at the ocean floor as discovered 30 years ago at Earth deep-sea hydrothermal vents.

Another potential player in Europa's habitability is its surface, which is exposed to the harshest radiation environment in the Solar System [17, 19, 67, 68]. This very high level of radiation may modify or alter the development of sub-surface prebiotic chemistry, and dissociate organic molecules at the surface. But it may also provide an energy source, via the storage of chemical free energy in the irradiation products. 


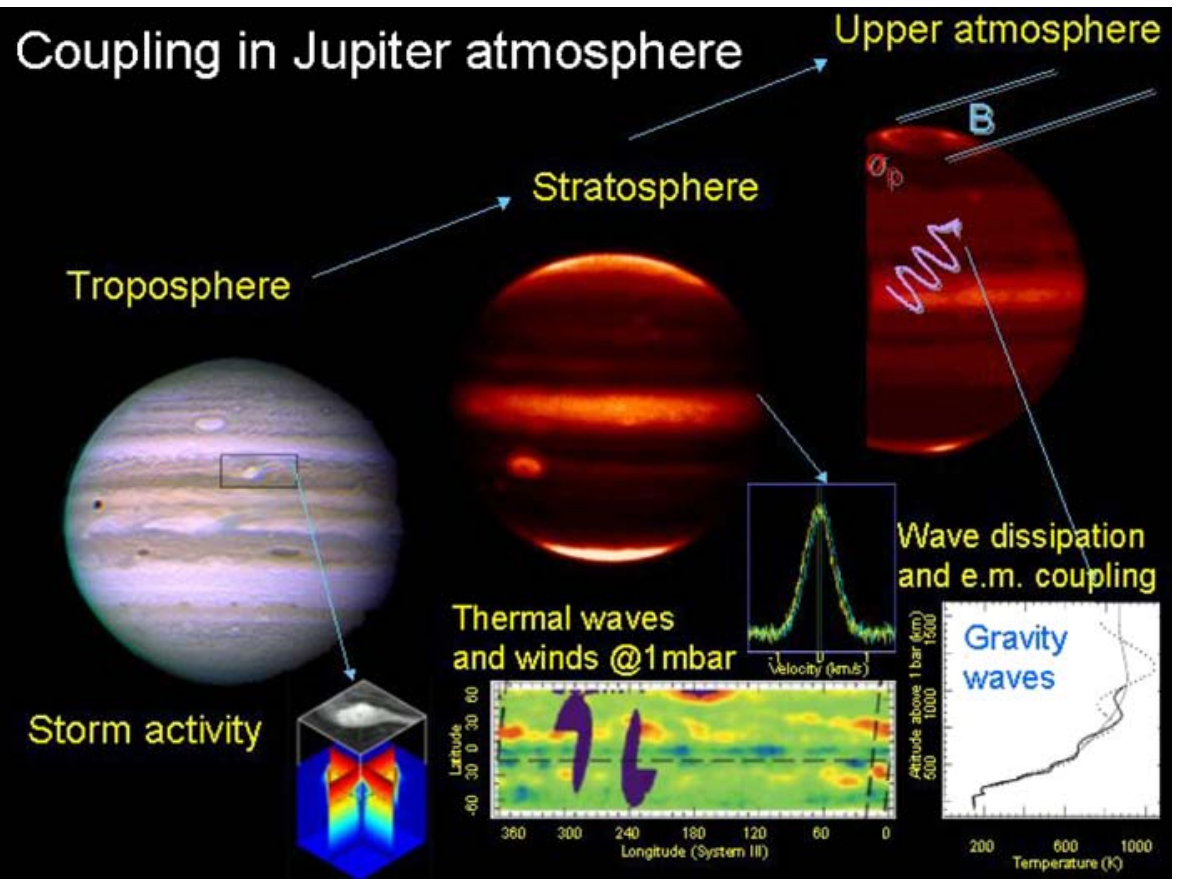

Fig. 6 The three layers of the Jupiter atmosphere studied by LAPLACE: (1) The dynamics of the troposphere will be studied with special emphasis on the source of vorticity, and its relation to global circulation. Storm activity is suspected of being a major source of vorticity, which is an important ingredient in the atmospheric circulation, and can also be the source of gravity waves dissipating in the upper atmosphere. (2) The circulation of the stratosphere (image at the cloud top $\sim 100$ mbar level, as observed at $3.5 \mu$; VLT/ISAAC image, P. Drossart, private communication) is poorly known. Doppler resolved spectroscopy can measure wind velocity at the limb and thermal waves can be imaged in the mid-IR and studied in the long term to observe the spatial and temporal scales (Cassini/CIRS observation). (3) Dynamical changes in the upper atmosphere can be studied by observing $\mathrm{H}_{3}^{+}$or $\mathrm{CH}_{4}$ emissions in the mid-IR to visual range (VLT/ISAAC image at $3.2 \mathrm{micron}$, P. Drossart, private communication), indicating connections to the magnetosphere in the auroral regions or to internal phenomena

\subsubsection{Assessing Europa's habitability}

Thanks to its Europa Orbiter, LAPLACE will assess all the elements that play a role in Europa's potential habitability, focusing on the characterization of its ocean, its sea-floor, and of its surface.

Looking for Europa's ocean After the Galileo mission around Jupiter, both geological evidences (tectonic features and surface composition) and geophysical data (induced magnetic field and gravity field) are strongly in favour of the existence of a deep liquid layer below an icy crust [13, 31, 44, 65]. However, although very probable, the existence of a liquid internal ocean is still not proven. If it exists, its depth is still unknown. It could be very close to the surface $(1-2 \mathrm{~km})$ or much deeper (20 $\mathrm{km}$ or more). The most robust method to prove the existence of the ocean is to use an orbiter on which accurate gravimetric and altimetric measurements can be achieved. Indeed, the presence of an ocean strongly modifies both the value of the 
Fig. 7 Present habitability of Europa. Possible locations of present life and biosignatures have been plotted as a function of depth. Habitability depends on physical and chemical constraints which are indicated on the right using colour scales (green: highly favourable; red: hostile). Numbers refer to possible interior structures described in Section 3.2

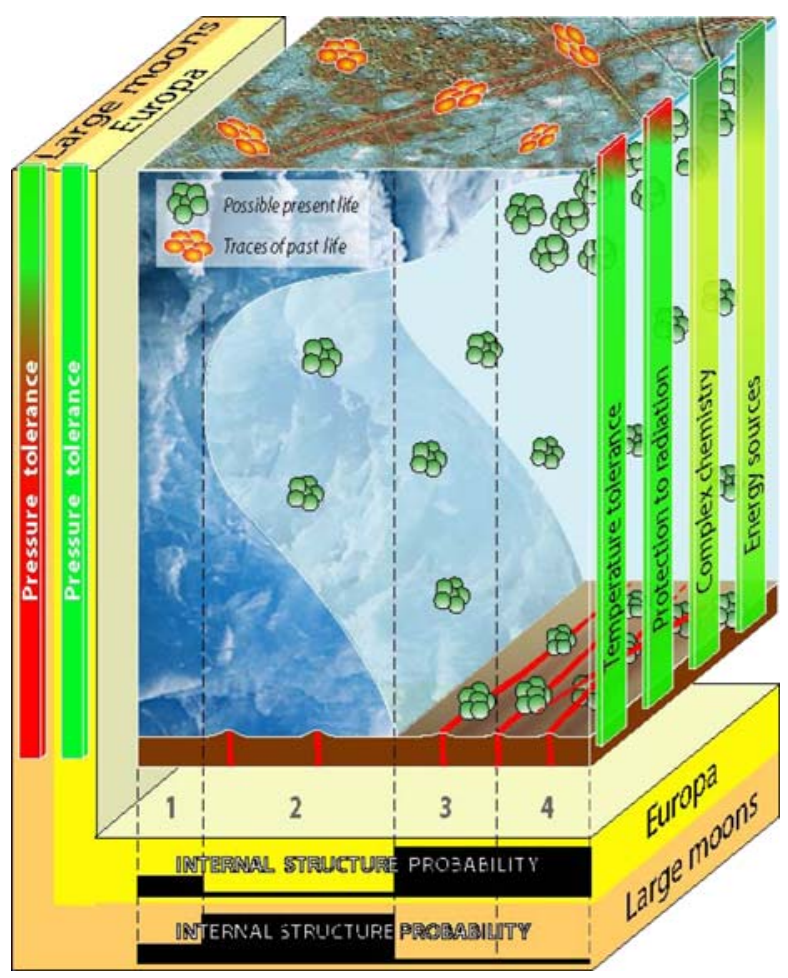

periodic Love number $\mathrm{k}_{2}$ and the amplitude of surface libration due to tidal forcing $[82,83]$. Such measurements were not achieved with Galileo because of orbital and instrumental constraints (only flybys, no altimeter), but may become possible with the LAPLACE Europa orbiter. If the gravity potential surface is obtained at degree 2 using a precise navigation system, it is possible to estimate the $\mathrm{k}_{2}$ love number value and prove the existence (or absence) of Europa's ocean [14]. If in addition an altimeter is added to the system, it is possible to have a direct and independent proof of the existence of the liquid layer by measuring the amplitude of surface motions due to tidal forcing. The in-situ, gravimetric and altimetric measurements at Europa will be supplemented by ultra-precise VLBI and Doppler tracking of the LAPLACE spacecraft by the Earth-based network of radio telescopes.

Characterization of the liquid layer is more challenging. It requires the determination of its depth, thickness and composition. The interface between the icy crust and the ocean is expected to be relatively flat [83]: since it corresponds to a thermodynamical equilibrium between a solid ice and its liquid counterpart, temperature and pressure along the interface must be almost constants, which impedes significant depth variations. Furthermore, the flat topography of Europa is not in favor of large roots of ice below high mountains as we see on Earth. Two specific instruments can be considered for providing direct constraints on the icy crust thickness. First, a Ground Penetrating Radar (GPR) seems very attractive. If the icy crust is relatively thin, this instrument will provide a global mapping of the ice I/liquid interface, provided that the crust is not thicker than the penetration depth, which is not guaranteed [16]. A 
GPR is thus challenging but risky. A more robust instrument is a seismometer. If the final mission can afford a lander or a penetrator, a seismometer will provide without ambiguity the position of the ocean interface. To our knowledge, this is the only direct measurement of the position of the ocean that can be envisaged and which will provide information regardless of the internal structure. A third technique is based on interpretation of topography and gravity field at different degrees. It requires a laser or radar altimeter in addition to the determination of the gravity field by radio measurements, including VLBI, two-way Doppler and delta-doppler ranging techniques.

Mapping Europa's seafloor If an internal ocean exists, Europa probably represents a single case, in which liquid water is in contact with a silicate core (Fig. 7). These unique conditions on Europa allow water-rock interactions, especially if any volcanism exists [80]. The determination of the topography and/or mass anomalies at the silicate core/liquid interface would provide hints on whether volcanism exists or has existed.

We must keep in mind that this task is a very difficult one mainly because the presence of the icy crust above the Europa's liquid layer decouples the surface topography from the ocean floor. But some constraints will be inferred from the accurate altimetry measurements and the precise determination of the gravity field at different altitudes. Moreover, magneto-meter measurements will be useful since the constraints provided on the induced magnetic field of Europa will help to define the depth of the magnetic source.

Small scale features won't be detectable on the silicate sea-floor, but medium-scale features $(200 \mathrm{~km})$ such as large volcanoes or long ridges will probably be detected if a microgradiometer is embarked on the Europa Orbiter. All these data (gravimetry, altimetry, magnetometry) will finally be used in models of the internal structure that will eventually give constraints on the ocean floor topography.

It should be stressed that, although difficult, the determination of the ocean floor topography and the state of iron core as well have strong astrobiological implications, and can only be done if the mission design can provide very precise navigation measurements, and both altimetric, gravity and magnetometric measurements with very high accuracy.

Characterizing its surface dynamics and chemistry: surface composition and chemistry Galileo has proven that the surface of Europa is not made of pure water ice [12, 53, 54]. Whatever the origin of the non-water components was, either endogenous or exogenous, their characterization is important. The NIMS (Near Infrared Mapping Spectrometer) onboard the Galileo mission showed that composition is different along some geological features. Although there is still some controversy on the nature of non-ice components, compositional mapping of the interesting areas of the surface must be improved in the future with a higher spatial and spectral resolution infrared mapping spectrometer. With a field of view of $0.5 \mathrm{mrad}$, a resolution of $1 \mathrm{~km} /$ pixel on the region where non-icy material has been discovered would be obtained at $200 \mathrm{~km}$ altitude.

In addition to a NIR mapping spectrometer, the main neutral species as well as many ion species of Europa's atmosphere and ionosphere can be easily measured with a mass spectrometer and dust analyzer on an orbiter at $200 \mathrm{~km}$. Moreover, 
collecting dust expelled from the surface of Europa, and analysing their composition will constrain the origins of building blocks that took part to its formation (via the $\mathrm{D}: \mathrm{H}$ ratio in ice if we assume that surface altering can be quantified with LAPLACE) and the thermodynamical conditions prevailing during Europa's formation (via the abundance of volatile species like $\mathrm{CH}_{4}, \mathrm{CO}_{2}, \mathrm{NH}_{3}$ ).

The composition of the surface, and even of the subsurface ocean (if connections between the interior and the surface exist or have existed) can then be inferred. However, it should be kept in mind that the surface environment has very extreme conditions, and materials from interior may be altered by depressurization, abrupt change in temperature, and particularly exposure to high radiation environment. These alteration processes must be studied, in particular by inferring the effect of radiation and energetic particles.

Studying its global surface morphology \& dynamics The surface of Europa is very young: both crater counting and estimation of erosion rates by sputtering (constrained by the measurements of $\mathrm{H}_{2} \mathrm{O}$ escape rates by Galileo) suggest an age of Europa's surface between ten millions and one billion years. This surface presents very intriguing tectonic and volcanic features (Fig. 8). The size and geometry of linear features suggest surface motions enabled by ice-crusted water or soft ice close to the surface. Impact features suggest the existence of a low viscosity layer at about 5 to $20 \mathrm{~km}$ in depth that may be either a liquid layer or a soft ice layer. For some of these intriguing features, Galileo also provided topographic estimates using the shadows of the elevated terrains. Interpretations of these features gave some of the strongest arguments in favour of a deep liquid ocean below the icy crust. That is why one of

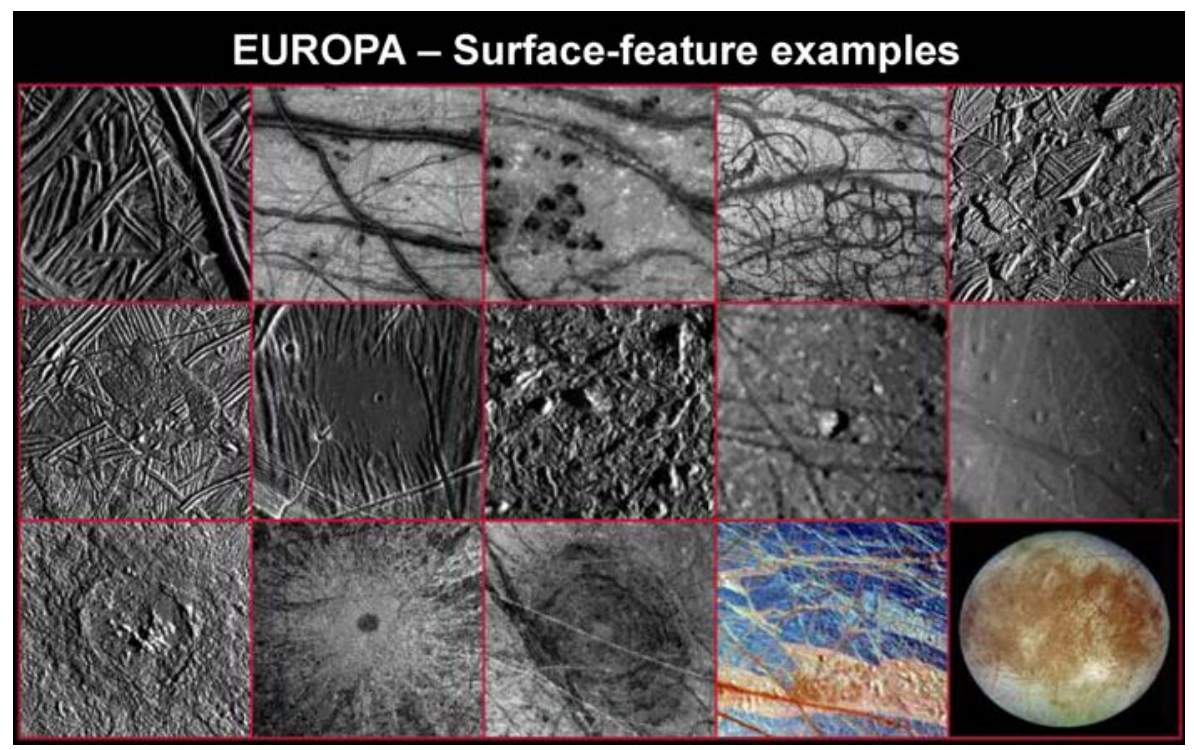

Fig. 8 Surface characteristics of Europa observed with Galileo. Many of these features strongly suggest the existence of a deep liquid layer under the icy crust 
the major goals of the LAPLACE Europa orbiter will be to study surface structures in more detail was achieved by Galileo.

Both a high-resolution camera, and a laser altimeter will be put on the Europa orbiter. The full coverage of the surface from a low orbit will permit the acquisition of high resolution images of every tectonic/volcanic feature. Laser altimetry measurements will bring new and valuable information on the topography, which will be of considerable importance for understanding geologic processes, and thus constraining the coupling between the putative ocean and the icy crust.

Finally, such high-resolution analysis of the surface will help to define astrobiologically interesting landing sites for future space missions.

Unraveling surface/exosphere/magnetosphere interactions The atmosphere of Europa is produced mainly from irradiation of its exposed outer surface by magnetospheric plasma (mainly sulphur and oxygen ions from Io) and UV photons. Its main component, molecular oxygen, has been indirectly observed by HST. Oxidized constituents produced by irradiation may also provide key components for life support within the subsurface ocean. The atmospheric composition of Europa is determined by both the water and oxygen photochemistry in the near-surface region, escape of suprathermal oxygen and water into the Jovian magnetosphere and exchange of radiolytic water products with the porous regolith.

Water ice is transformed into its gaseous state by sputtering processes and sublimation. Generally the water sublimation rate should be extremely low. However, around the dayside equator the observed increase of the sublimation rate of at least four orders of magnitudes than expected means that sublimation could be a major process for water vapour production. Cryovolcanic activity is another possible source of water vapour around satellites. "Boiling" water which may also exist due to thermal flexing and cracks in the surface will result in a highly increased supply of water vapour to the atmosphere and thus source regions will be easily detectable.

Europa's atmosphere interacts with the magnetospheric plasma by a large variety of processes including sputtering of the surface and exosphere and resurfacing due to intense bombardments by energetic particles. Energetic ions and electrons are the principal chemical agents in layers close to the surface of moons that could alter species originating from the deep interior and migrating to the surface.

LAPLACE will monitor the incoming and reflected radiations, the structure and composition of the exosphere and ionosphere, surface composition to establish the net production, loss, and exchange of key chemical species between the magnetosphere, exosphere/ionosphere, surface and subsurface.

Characterization of potential environments and search for biosignatures If Europa is (or was) habitable, the next exploration step should be the search for any signature of life that the environment is able to sustain. Remote detection of reliable biosignatures is very difficult, and in situ exploration is clearly preferable. That is why the mission study includes as an option a surface module (SE) that will allow to obtain environmental data (radiation, $\mathrm{pH}, \mathrm{eH}, \mathrm{T}$, redox, chemistry) of the surface, and possibly of the near-surface. Geological evidences indicate that a link between the surface and the aqueous layer could have existed [30]. Therefore, examination of endogenous materials, deep enough to avoid alteration effects by Jupiter's radiation, may reveal some clues of the aqueous layer properties. 
2.4 From science goals to mission constraints and measurement requirements

Our science goals address four different mission targets in the Jupiter system: Europa, the satellite system, Jupiter's magnetosphere, and the planetary atmosphere. Through a careful analysis of the measurements to be performed, we have derived specific requirements for the measurements we need to perform on each of these objects, and specific constraints on the mission profile of LAPLACE. They are summarized in the following four tables, which represent the traceability matrix of the LAPLACE mission.

\section{LAPLACE mission profile}

\subsection{Platform and orbit requirements}

LAPLACE will deploy an observing system to perform the key observations needed at each target. As shown in Fig. 9, this observing system will use a hierarchy of observing posts or platforms and strongly link Earth-based and space-based observations.

Earth-based and Earth-bound observations will be conducted on two distinctive sets of targets: the LAPLACE mission objects (Jupiter, Europa, other components of the jovian system and their environment) and the LAPLACE mission spacecraft.

The former will help to prepare the mission, and then place space observations in a broader context during the mission itself, sometimes even providing observational data not achievable by space-based mission instruments. These astronomical observations will be conducted in a broad range of wavelengths, from high-energy domain to low radio frequencies.

The latter will include VLBI and Doppler tracking of the spacecraft aimed at achieving very high accuracy of trajectory characterization of all mission spacecraft in various phases of their operations. In addition, the radio telescopes involved in VLBI tracking, as well as the Square Kilometre Array (SKA) which will be operational during the LAPLACE orbital mission, will provide a backup "eavesdropping" support to the nominal mission communication scenario enabling receipt of low datarate signals during critical and high scientific value events of the mission.

The space segment will observe the targets at progressively closer distances: from interplanetary orbit, during the approach to Jupiter; then from Jupiter orbit; finally from satellite orbits, first at Europa, our priority target, and possibly at Ganymede. If resources and technology allow, a Europa surface element could be deployed. It will need to be an integrated, multi-platform observing system of our four target. Ideally, it should include the coordinated operation of three orbiters:

The Jupiter Europa Orbiter (JEO), a nadir-pointing platform optimized for global coverage of the surface, subsurface and internal structure of Europa, will perform a multi-month mapping of Europa before impacting its surface.

The Jupiter Planetary Orbiter (JPO), a three-axis stabilized platform optimized for remote sensing observations of distant objects, will observe Jupiter, its satellites and probe their internal structures. 


\section{LAPLACE MISSION TRACEABILITY MATRIX}

a

I. Chamacterize Europa as a planetary object and a potential habitat

\begin{tabular}{|c|c|c|c|}
\hline Scientific objectives & Required measurements & Enabling instrumentation & $\begin{array}{l}\text { Contraints } \\
\text { on mission }\end{array}$ \\
\hline $\begin{array}{l}\text { Existence \& characterization } \\
\text { of a sub-surface ocean }\end{array}$ & 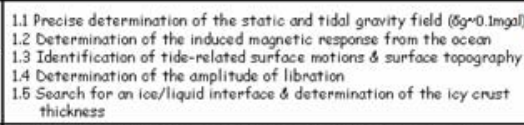 & 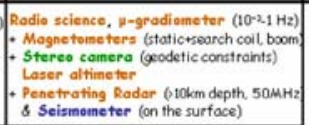 & \multirow{7}{*}{ 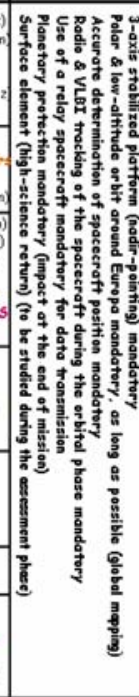 } \\
\hline $\begin{array}{l}2 . \\
\text { Silicate/oceon } \\
\text { surface topography }\end{array}$ & $\begin{array}{l}2.1 \text { Determination of the topography and/or mass anomalies at the } \\
\text { sticate core/liquid interface } \\
\text { 2.2 Search for correlation between surfoce and sub-surface structures } \\
2.3 \text { Characterization of global and local heat flows }\end{array}$ & 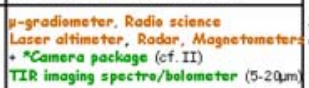 & \\
\hline $\begin{array}{l}\text { 3. } \\
\text { Mineral and chemical } \\
\text { composition of the surfoce } \\
\text { from orbit }\end{array}$ & 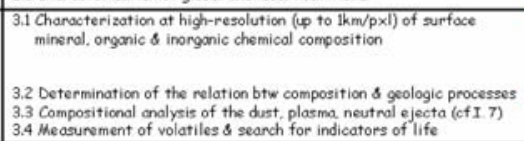 & 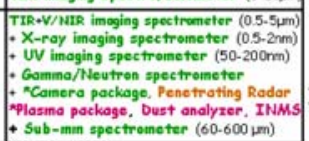 & \\
\hline $\begin{array}{l}\text { 4. In--situ } \\
\text { choracterization ond search } \\
\text { for biosignatures on } \\
\text { observable } \\
\text { potential environments }\end{array}$ & $\begin{array}{l}\text { 4.1 Me asure key habitability parameters (physics \& chemistry)! } \\
\text { T. pH. Eh, electrical conductivity. rodiation conditions, redox couples } \\
\text { 4.2 Measurement of organic molecules related to past and/or extant life } \\
\text { 4.3 Measurement of inorganic signatures of life } \\
\text { 4.4 Search for biologioal / fosall structures. }\end{array}$ & $\begin{array}{l}\text { On the surface } \\
\text { Penetrator: environenental } \\
\text { multi-sensors } \\
\text { mapenetrator: chemistry \& mineralogy } \\
\text { experiments }\end{array}$ & \\
\hline $\begin{array}{l}\text { 5. } \\
\text { Global surfoce } \\
\text { orphology \& dynomics }\end{array}$ & $\begin{array}{l}5.1 \text { Characterization of the surfoce properties } \& \text { topography } \\
5.2 \text { Identification of tectonic, volcanic, } A \text { impoct features } \\
5.3 \text { Search for sites of recent geological activity } \\
5.4 \text { Determination of interesting sites for a future lander }\end{array}$ & 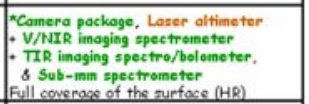 & \\
\hline $\begin{array}{l}\text { 6. Constraints on the deep } \\
\text { internal structure \& dynamics }\end{array}$ & $\begin{array}{l}\text { 6.1 Determination of the state of Europa's iron cone } \\
6.2 \text { Detection of sub-solidus convection in Europac's cone }\end{array}$ & $\begin{array}{l}\text { Radio science, p-gradionneter } \\
\text { + Magnetoineters + Seismoineters }\end{array}$ & \\
\hline $\begin{array}{l}7 . \\
\text { Exosphere/Mognetosphere } \\
\text { interoctions }\end{array}$ & $\begin{array}{l}\text { 7.1 Characterization of the rodiation environment (cf. IIII } 3 \text { ) } \\
7,2 \text { Characterization of the sputtering s sublimation processes } \\
7,3 \text { Study of the ionosphere } \& \text { neutral atmosphere } \\
7,4 \text { Study of escope rates } \& \text { exogenic deposition }\end{array}$ & 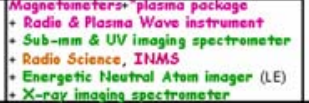 & \\
\hline
\end{tabular}

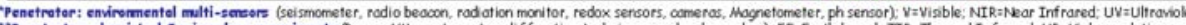

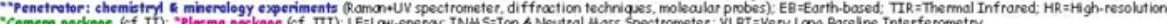
Cemera package (cf. If); "Plasma pockage (cf. III); LE=Low-energy, INMS:Ion \& Neutral Mass Spectrometer; VL.BI = Very Lang Baseline Interferometry

b

Study the Jovian "satellites system and their connection to the population of minor bodies in the Solar System * Europa is discussed in I.

\begin{tabular}{|c|c|c|c|}
\hline $\begin{array}{l}\text { Internal structure } \\
\text { long-terin evolution } \\
\text { of the Galliean satellites }\end{array}$ & 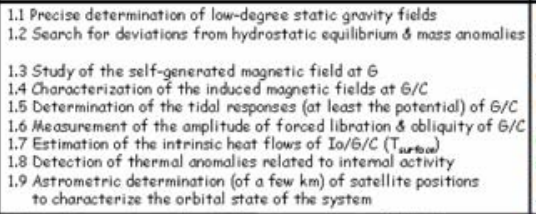 & 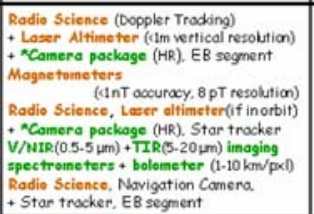 & \multirow{4}{*}{ 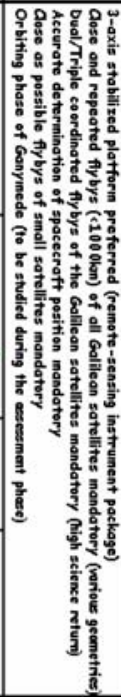 } \\
\hline $\begin{array}{l}2 . \\
\text { Physical characteristics. } \\
\text { composition \& geolegy } \\
\text { of the surfaces } \\
\text { of the Golilean satellites }\end{array}$ & $\begin{array}{l}\text { 2.1 Geologic mapping with significant improvement of global coverage } \\
\text { 2.2 Topogrophic mopping of large fractions of the satellites' surfaces } \\
\text { 2.3 Search for past and present geologic activity from limb scans } \\
\text { thermal anomalies and image comparison } \\
\text { 2.4 Monitoring of Io's volcanic \& themnal activity on day- \& nightside } \\
\text { 2.5 Determination of global \& regicnal surface ages (cratering record) } \\
\text { 2.6 Characterization of the non-ice components and search for organic } \\
\text { compounds, in relation to geologic and tectonic features } \\
\text { 2.7 Determination of elemental cbundances from spectroscopy } \\
\text { 2.8 Mapping of surface alterations due to rodiation environment }\end{array}$ & 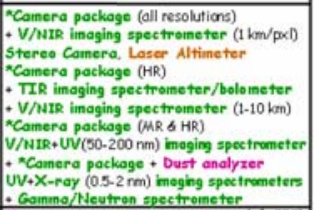 & \\
\hline $\begin{array}{l}\text { Interactions } \\
\text { Ietween magnetosphere, } \\
\text { exosphere \& surface }\end{array}$ & $\begin{array}{l}2.1 \text { Study of the induced magnetospheres of } \mathrm{Io} / \mathrm{C} \\
2.2 \text { Detailed study of the intrinsic mognetosphere of } 6 \\
2.3 \text { Study of the ionospheres and exospheres of the satellites } \\
2.4 \text { Study of escape rates and exogenic depositions }\end{array}$ & 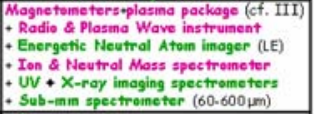 & \\
\hline $\begin{array}{l}\text { Evelution } \\
\text { of the Jovian systein } \\
\text { by investigating } \\
\text { the sinall regular inner } \\
\text { satellites, } \\
\text { the ring systein, } \\
\text { the irregular outer satellites. }\end{array}$ & 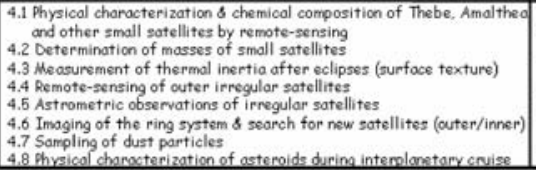 & 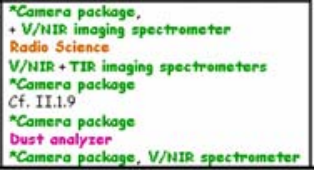 & \\
\hline
\end{tabular}

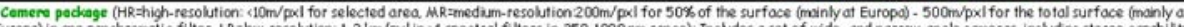
Europa) in ane penchromatic filter, $L R=$ bw-resolution $1-2 \mathrm{~km} / \mathrm{pocl}$ in $>4$ spectral filters in $350-1000 \mathrm{rem}$ range): Includes a set of wide-and narrow-angle cameras, including stereo copability.

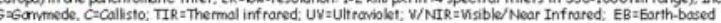


C

III. Study the Jovian magnetodisk/magnetosphere

\begin{tabular}{|c|c|c|c|}
\hline Seientific objectives & Required measurements & Enabling instrumentation & $\begin{array}{l}\text { Contraints } \\
\text { on mission }\end{array}$ \\
\hline \multicolumn{3}{|l|}{$\begin{array}{l}\text { Jupiter as a } \\
\text { fast magnetic rotator }\end{array}$} & \multirow{6}{*}{ 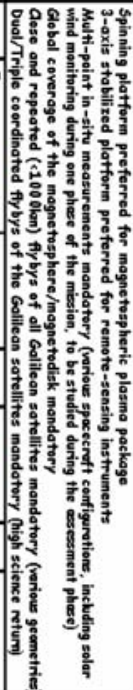 } \\
\hline $\begin{array}{l}1 . \\
\text { Magnetodisk structure, } \\
\text { dissipation of } \\
\text { rotational energy. } \\
\text { transfer of } \\
\text { angular mo inentun }\end{array}$ & $\begin{array}{l}\text { 1.1 Chanacterization of the } 30 \text { properties of the magnetodisk } \\
\text { 1.2 Analysis of the influence of the various plasma sources } \\
\text { on its structure (including composition measurements) } \\
\text { 1.3 Response to mass looding variability (Ionian space weather) } \\
\text { 1.4 Characterization of the different modes of tronsport } \\
\text { 1.5 Determination of the plasma loss processes }\end{array}$ & $\begin{array}{l}\text { Magnetometers (statictsearch coil boom) } \\
\text { * "plasma package (all) } \\
\text { - Longinuir probe (spacecraft potential) } \\
\text { + Radio \& Plasma Wave instrument } \\
\text { - Energetic Neutral Aton imager } \\
\text { + X-roy / UV imaging spectrometers } \\
\text { (0.18-12rm/ 50-200nm, tori emissions) } \\
\text { + EB segment (VIS/UV telescopes) }\end{array}$ & \\
\hline $\begin{array}{l}2 . \\
\text { Global energy regulation } \\
\text { in a complex magnetosphere }\end{array}$ & 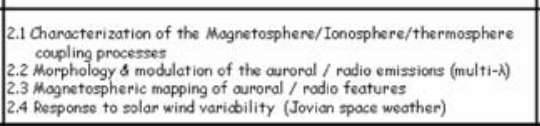 & 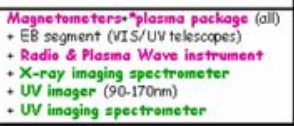 & \\
\hline $\begin{array}{l}\text { 3. } \\
\text { Satellites/Magnetosphere } \\
\text { interactions }\end{array}$ & $\begin{array}{l}\text { 3.1 Charocterization of the local electrodynamics interoction } \\
3.2 \text { Observations of the moon auroral magnetic footprints } \\
3.3 \text { Study of pick-up \& change-exchange processes in plasma/neutral tori } \\
3.4 \text { Analysis of plasma/surface sputtering processes }\end{array}$ & 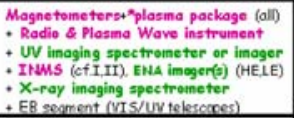 & \\
\hline \multicolumn{3}{|l|}{$\begin{array}{l}\text { Jupiter as a } \\
\text { giant porticle occelerotor }\end{array}$} & \\
\hline $\begin{array}{l}\text { Origin \& effects } \\
\text { of its harsh rodiotion } \\
\text { environunent }\end{array}$ & $\begin{array}{l}\text { 4.1 Characterisation of high-energy particle properties } \\
\text { 4.2 Identification of high-energy particle accelenation processes } \\
\text { 4.3 Determination of high-energy particle loss processes } \\
\text { 4.4 Analysis of moon micro-signatures to quantify fundamental processes } \\
\text { 4.5 Analysis of high-energy electrons synchrotron emissions }\end{array}$ & $\begin{array}{l}\text { Magnetometerst" plasma pockage (alli) } \\
\text { - Rodiation monitor (Up to } 100 \mathrm{MeV} \text { ) } \\
\text { EB segment (VLA LOFAR) }\end{array}$ & \\
\hline
\end{tabular}

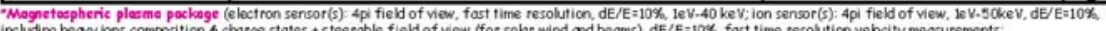

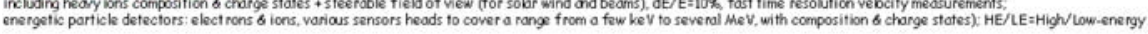

d

IV. Study the Jovian atmosphere and interior

\begin{tabular}{|c|c|c|c|}
\hline Seientific objectives & Required measurements & Enabling instrumentation & $\begin{array}{l}\text { Contraints } \\
\text { on mission }\end{array}$ \\
\hline \multicolumn{4}{|l|}{$\begin{array}{l}\text { Dyma inics \& coupling } \\
\text { in Jupiter's at mosphere }\end{array}$} \\
\hline $\begin{array}{l}1 . \\
\text { Stratospheric structure, } \\
\text { circulation \& composition }\end{array}$ & $\begin{array}{l}\text { 1.1 Determination of the origin of water in the stratosphere } \& \text { of its role } \\
\text { in atmospheric chemistry \& dymamics } \\
1.2 \text { Characterization of latitudinal variations of stratospheric water to } \\
\text { constrain its origin } \\
1.2 \text { Characterization of the nature of the hydrocarbon chemistry } \\
1.3 \text { Determination of the composition of the primordial material from } \\
\text { which Jupiter formed } \\
1.4 \text { Observations of the dispersion of HCN in the atmosphers following } \\
\text { the SL-9 impacts } \\
\text { 1.5 Characterization of the strength of the vertical mixing in the } \\
\text { stratosphere }\end{array}$ & \begin{tabular}{|} 
Sub-imin spectroineter $(60-600, \mathrm{~m})$ \\
+ Cainera (multi-color) \\
+ *IR imaging spectroineter \\
+ EB segment (e.g-odaptative optics. \\
AUMA. Herschel)
\end{tabular} & \multirow{4}{*}{ 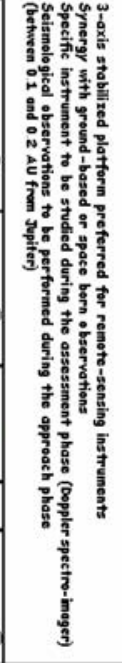 } \\
\hline $\begin{array}{l}\text { 3. } \\
\text { Therinospheric \& ienospheric } \\
\text { structure \& dynamics }\end{array}$ & $\begin{array}{l}\text { 3.1 Determination of the origins of high thermospheric temperatures } \\
\text { 3.2 Characterization of the vertical coupling in the atmosphere \& } \\
\text { of its drivers (EUV heating, ion drag or wave activity) } \\
\text { 3.3 Characterization of auroral emissions vaniations } \\
\text { 3.4 Characterization of ionospheric total electron densities } \& \text { variations } \\
\text { 3.5 Detection of } \mathrm{H} 3 \text {. at low latitudes }\end{array}$ & $\begin{array}{l}\text { PR inaging spectroineter } \\
X \text {-ray inaging spectroneter, } \\
+ \text { UV inaging spectronneter or inager } \\
+ \text { Rodio science } \\
+ \text { EB segment (IR) }\end{array}$ & \\
\hline \multicolumn{3}{|l|}{$\begin{array}{l}\text { Intemal strueture } \\
\text { \& composition of Jupiter }\end{array}$} & \\
\hline Formation of Jupiter & $\begin{array}{l}\text { 4.1 Qefermination of the presence of a cone at Jupiter } \\
4.2 \text { Characterization of the internal density profile of Jupiter } \\
4.3 \text { Determination of the extension of the metallic hydrogen envelope } \\
4.4 \text { Determination of the chemical, isotopic } \& \text { elemental composition } \\
\text { of Jupiter's atmosphere } \\
4.4 \text { Characterization of the behoviour of matter under extreme condition } \\
\text { (H \& He equations of state) }\end{array}$ & $\begin{array}{l}\text { boppler spectro-imager } \\
\text { (430-520 rm, seismobgical tectniques for } \\
\text { acoustic wores detection, } \\
\text { N.B. waves at higher harmonics con also be } \\
\text { used to sound the upper troposphere) } \\
\text { - EB segment (Laser Mega Joule experiments? }\end{array}$ & \\
\hline
\end{tabular}

"IR imagim spectrometer (integrated IR instrument including multicharnels $(\omega 20)$ from 2 to $20 \mu m$, with 3 chanels for imaging spectroscopy: mid-IR for Temperature/Pressure, NIR at low-resolution for cloud and continum retrieval, NIR at high-resolution for $\mathrm{H} 3 *$ ): IR=IInfrared, NIR =Near-Inf rared. EB = Earth-based 


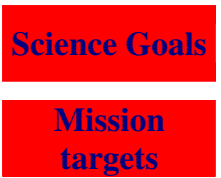

Eart-based observations

Interplanetary orbit

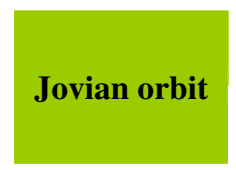

Satellite orbits

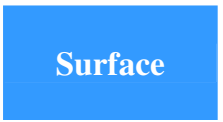

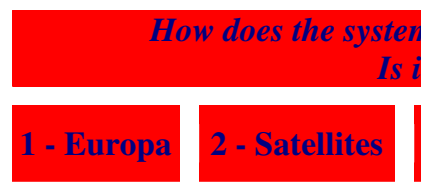

Is it habitable?

3 - Magnetosphere

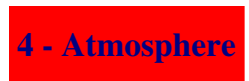

JWST, ALMA, VLT, ELT's, radioastronomy

VLBI (includes interaction w. space segment)
V/E fly-by science, Seismology, gravity, Dust streams, radio emissions, electron beams, etc,... (IP)
Jupiter Magnetosphere Orbiter (JMO)

Jupiter Planetary Orbiter (JPO)

\section{JEO}

Ganymede orbiter ? (GO)

Surface

element ?

(SE)

Fig. 9 Schematic showing how the different science goals and targets of the mission will be addressed by a comprehensive observing system including a ground-based segment and a 3-platform spacebased segment progressively deployed on "strategic" orbits and locations inside the jovian world

The Jupiter Magnetospheric Orbiter (JMO), a spinning platform optimized for in-situ fields and particles measurements, will monitor the magnetosphere, the moons exospheres and their interactions. These platforms will operate in strong operational synergy, as the JPO will be used as a relay satellite for the JEO data during the critical period of its operations in Europa orbit. They will also be in strong scientific synergy: multi-point studies of the magnetosphere; combinations of close, high-resolution observations of Europa by the JEO with more distant, synoptic views by the JPO; associations between ENA or radio imaging of various magnetospheric particle populations by one platform with in situ characterizations of these same populations by another platform.

Two expanded options, to be studied during the assessment phase, have been considered:

a Europa Surface Element (SE) would represent considerable added value to the exobiology and geosciences objectives of the mission. Its deployment represents a major scientific and technological challenge. It would require the definition of an efficient strategy for the timely selection of a landing site with a high exobiological and geological potential. A possible stand-alone Surface Element, to be closely coordinated with LAPLACE as an additional segment of this mission, is considered and studied by Roscosmos. It is described in the Appendix. 
a Ganymede Orbiter (GO) could be the final configuration of the JPO, if DeltaV resources allow. Such an orbiter would also add considerably to the mission science, as it would provide the basis for an in-depth comparative study of the main two differentiated icy satellites among the Galilean system: Ganymede and Europa.

\subsection{Launcher requirements and possible collaborative scenarios}

We have identified at least three possible scenarios to launch and deploy this observing system. The basic mission architecture-a multi-platform, multi-target integrated mission-clearly speaks in favour of a broad international collaboration. This is what our two preferred scenarios (1 and 2) assume. These scenarios will have to be explored and consolidated with the international partners by means of a joint assessment study. One stand-alone ESA scenario (3) is also proposed.

Scenario 1 Two medium capacity launchers (Soyuz/Fregat, HIIA and upgrades, Atlas $\mathrm{V}-5, \ldots)$

- $\mathrm{JPO}+\mathrm{JMO}$ on one launch

- JEO+(optional) SE on the second launch.

Scenario 2 One heavy launcher (Ariane 5, Atlas 5-5...) with the JEO +1 jovian orbiter (combining JPO and JMO). Its capacity for carrying SE (with JEO) or separate JPO and JMO spacecraft needs to be investigated during the assessment study.

Scenario 3 One Soyuz/Fregat launch (ESA-only mission) with two s/c: JPO/JMO and JEO. This more constrained scenario will address more limited but more focused objectives, mainly Europa.

\subsection{Trajectories and $\Delta^{\Delta}$ budgets}

Figure 10 illustrates our basic mission timeline, showing in the top panel how each platform moves from orbit to orbit with time. Five mission phases can be distinguished, for which the assessment will have to find the best possible orbits, trajectories and maneuvres, to optimize the science and minimize the total radiation dose and $\Delta^{\Delta}$ budgets. Phase 0 is the interplanetary trajectory. In all studied scenarios we suggest a 6-year long Venus-Earth-Earth swing-by trajectory with good launch opportunities in January 2017, March 2020 and June 2023. For Jupiter Orbit Insertion (JOI), the Jupiter capture strategy relies on an Io gravity assisted capture with a perijove at $4 \mathrm{Rj}$ and a first apojove at $\sim 400 \mathrm{Rj}$, which is a good compromise between $\Delta^{\Delta}$ cost and radiation dose. A Perijove Raising Manoeuvre (to about $13 \mathrm{Rj}$ ) is performed at the first apojove, after which the spacecraft are separated (JPO from JMO in scenario 1, JPO from JEO in scenarios 2 and 3). During phase 1, each spacecraft then starts its own Jovian tour, with multiple fly-bys of jovian moons (mainly Ganymede, Callisto and Europa) providing a unique opportunity for multispacecraft studies of the jovian system.

For phase 2, the JEO critical Europa mission takes place for a period of 60 to 90 day, to be validated during the assessment phase. The JPO is placed on a 2:1 
Fig. 10 A generic timeline of mission phases for the different flight components and the different mission scenarios and trajectory geometries for phase 0,1 and 2

resonant orbit with Europa to serve as a relay satellite. For phase 3, the JPO moves to a safer orbit to reduce radiation doses and relays the JEO data to the Earth while performing multiple flybys of Ganymede and Callisto. JMO moves to a $\sim 15 \times 70 \mathrm{Rj}$ equatorial orbit to explore the middle magnetosphere and perform multiple satellite fly-bys.

\subsubsection{Special requirements}

The overall scientific optimisation of the Jovian tours of the different spacecraft will have to be done taking into account the two main special requirements affecting the mission: keeping the radiation doses received by the three spacecraft below an acceptable level, and fulfilling Planetary Protection requirements. The severe radiation environment forces one to optimise the spacecraft trajectories and flight system radiation tolerance such that the radiation doses remain acceptable. This affects the $\Delta^{\Delta}$ budget, the shielding mass to be considered to meet a given radiation hardening level for platform equipment and science instruments, and the spacecraft lifetime in operational orbit. This trade-off at mission/system level will have to be performed during the assessment phase. The Planetary Protection requirements of COSPAR for Europa flybys, orbiters and landers, including bio-burden reduction, shall be applied in order to reduce the probability of inadvertent contamination of a europan ocean to less than $1 \times 10^{-4}$ per mission. In the case of JEO which impacts Europa at the end of its mission this will likely require bio-burden reduction. In the case of JPO and JMO, the option of reducing the probability of impacting Europa below $10^{-4}$ can relax or eliminate the need for contamination control and sterilization.

\subsection{Critical issues}

The tight mass budget limited by two medium sized (scenario 1) or one single heavy launcher (scenario 2), though much better than in scenario 3, requires stringent management of the mass allocations. The severe Jovian radiation environment and the remoteness from the Sun and the Earth also have implications for the mission and system design. Power must be provided with either high efficiency solar cells with concentrators or RTGs (an option that will have to be considered during assessment). The remoteness from the Earth and the limited power resources and lifetime of JEO poses a data volume and communications challenge which has been partly addressed by relaying data from JEO to JPO where it is stored and returned to Earth at a later time. This eliminates the need for a high gain antenna and high power transmitter on JEO mitigating the mass and power challenge for that spacecraft. Even so, relaying back to Earth the important data volume to be generated by the mission will require a specific strategy: design of on-board data compression and storage capacities with the appropriate robustness to radiations, data link capacities between spacecraft and to Earth, optimal use of Earth-based reception capacities, careful sequencing of observation phases to share the resources between all instruments and data acquisition and data relay sequences, will be major tasks for the assessment study phase. 


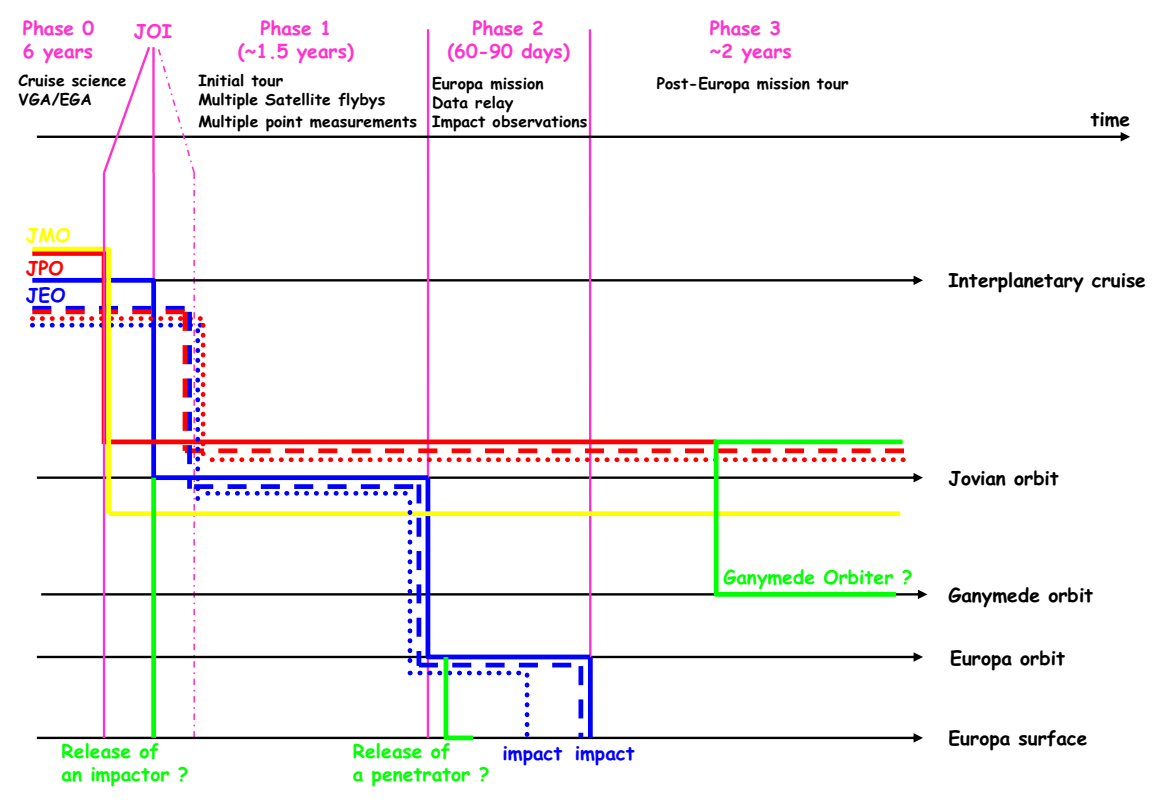

Scenario 1 Expanded options $=$ Scenario 2 "üü Scenario 3
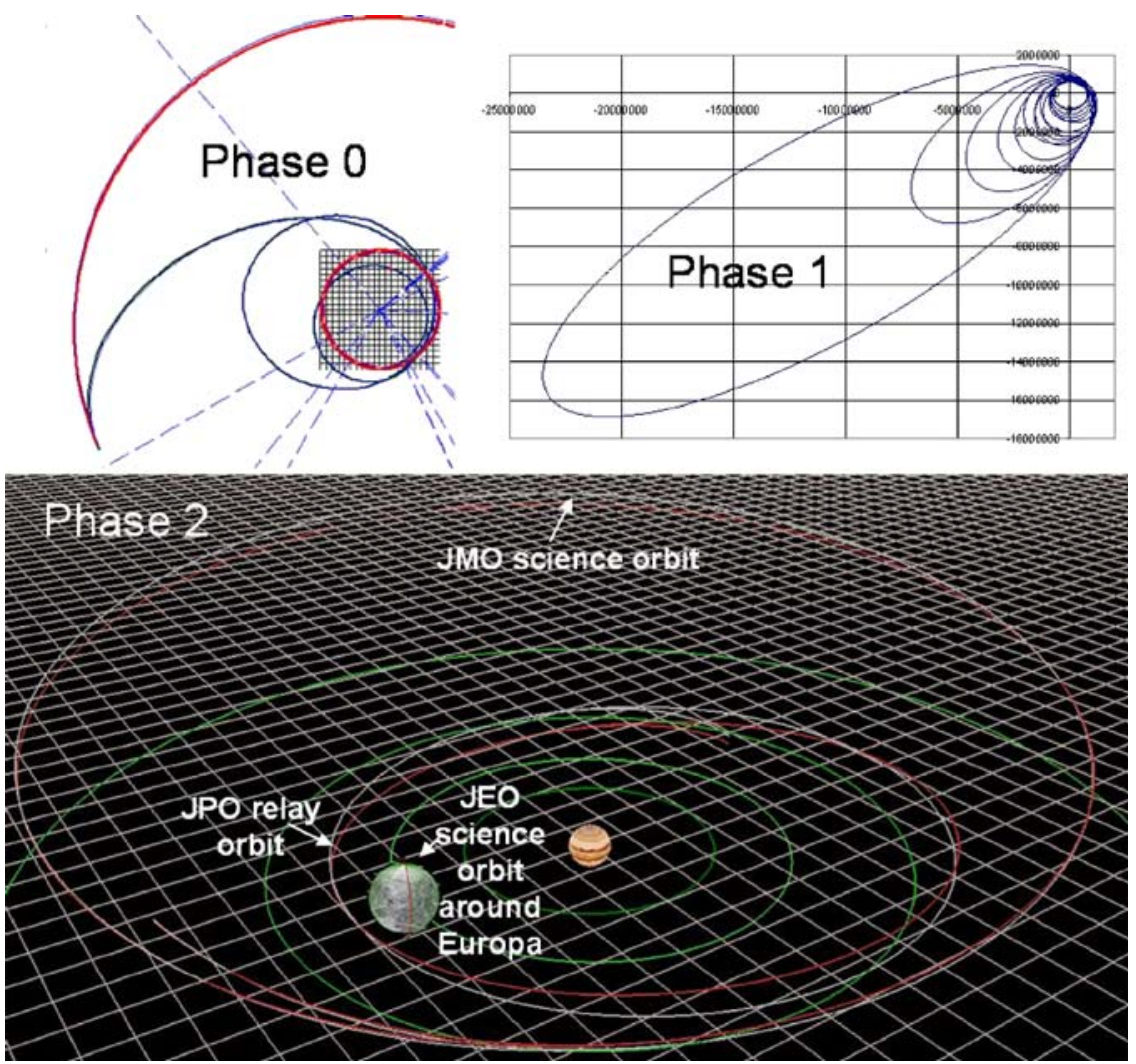


\section{Proposed payload instrument complement}

\subsection{Observation strategy}

LAPLACE addresses a broad spectrum of scientific objectives covering all themes of planetary sciences. Let us now propose a possible payload instrument complement to meet these objectives.

Three complementary categories of scientific investigations satisfy the measurement requirements of LAPLACE

- measurements of the main planetary fields of Europa, and to a lesser extent of the other satellites (gravity, magnetic field, geodesy and surface topography), including their response to tidal forcing, to retrieve the main characteristics of Europa's ocean and determine the internal structure of the different satellites,

- multi-spectral remote sensing measurements of the surfaces, atmospheres and gas tori, and possibly sub-surfaces of the different bodies of the Jupiter system, to retrieve their composition and dynamics and improve our understanding of the vertical coupling mechanisms between the different layers,

- in situ and remote sensing measurements of the plasmas, fields, energetic particles, gas and dust populations of Europa's exosphere and Jupiter's magnetosphere, to quantitatively understand the mutual interactions between the different bodies of the Jupiter system and its space environment.

\subsection{Overview of all payload elements}

An overview of the detailed list of instruments which address the objectives of LAPLACE is given in Table 1. The table shows how the instruments meet the stated scientific objectives listed in Section 2 and summarizes key resources for each of the instruments, in terms of mass, power and telemetry (when possible) budget. It also provides a synthesis in terms of pointing and alignment requirements and design constraints on mission elements. Instrument heritage is also shown, clearly revealing that the necessary experience and expertise is in place in order to design, build and fly such instrumentation. The resource figures rely mainly on state-of-the art and current technologies developed in European and foreign laboratories but the significant progress in instrument design and performances expected in the coming years will help in reducing the load on spacecraft resources (mass, power), as will be discussed later. In term of technology readiness level, most of these types of instrumentation have successfully flown on several planetary missions, including previous missions to Jupiter. A limited set of foreseen instruments are new and deserve feasibility studies during the assessment phase, as detailed in the following section.

\subsection{An example of a possible set of science payload elements}

Using the list of instruments from Table 1 as our starting point, and a realistic estimate of spacecraft resources for each mission scenario and each platform, we have identified a possible payload complement, given for illustrative purposes.

It consists of core scientific instruments, addressing high-priority science objectives, plus complementary instruments, for each scenario and mission elements. 
Table 1 Comprehensive list of instruments to be considered for LAPLACE, meeting the scientific objectives listed in Section 5 and showing key specifications, resources and design constraints

\begin{tabular}{|c|c|c|c|c|c|}
\hline \\
\hline Instrument & Science & Specifications & $\begin{array}{c}\text { Resources } \\
\text { (mass, power, TM) }\end{array}$ & sc & $\begin{array}{c}\text { Comments } \\
\text { Heritage/Design constraints }\end{array}$ \\
\hline $\begin{array}{l}\text { Laser altimeter } \\
\text { Magnetonneters } \\
\text { Miero-gradiometer (new) } \\
\text { Radio science experinent }\end{array}$ & $\begin{array}{l}\text { I.1,2,5; } \\
\text { II.1,2 } \\
\text { I.1,2,6,7: } \\
\text { II.1,3: III } \\
\text { I.1,2,6 } \\
\text { I.1,2,6,7: } \\
\text { II.1,4:IU.2,3 }\end{array}$ & $\begin{array}{l}1064 \mathrm{~nm}, \\
\mathrm{kHz} \text { shot rate } \\
\mathrm{Static}+\text { search coil } \\
+2-3 \mathrm{~m} \text { boom (TBD) } \\
\text { measurement band } \\
0.001-1 \mathrm{~Hz} \\
\text { Doppler tracking } \\
\text { transponder }\end{array}$ & $\begin{array}{l}12-15 \mathrm{~kg}, 34-67 \mathrm{~W} . \\
28 \mathrm{kbps} \text { at full res. } \\
\\
5 \mathrm{~kg}, 7 \mathrm{~W}, \\
30 \mathrm{kbits} / \mathrm{s} \\
2 / 3 \mathrm{~kg}, 2 \mathrm{~W} \text {. } \\
\mathrm{a} \mathrm{few} \mathrm{kb} / \mathrm{s} \text { (average) } \\
1 \mathrm{~kg}, 1 \mathrm{~W} \text {. } \\
\mathrm{TBO} \\
3 \mathrm{~kg}, 10 \mathrm{~W}\end{array}$ & $\begin{array}{c}3 \\
3 / 5 \\
3 \\
3\end{array}$ & $\begin{array}{l}\text { MARSIS on Mars Express } \\
\text { ESA CDF study } \\
\text { Electromagnetic cleonliness } \\
\text { Deployment } \\
\text { BELA on BepiColombo } \\
\text { Thermal, SA } \\
\text { MAG on Cassini, Venus Express } \\
\text { Magnetic cleanliness, Thermal } \\
\text { Stabilization required } \\
\text { Quiet vibration environment } \\
\text { Ultra-Stabilized Oscillator } \\
\text { Ground ontennas }\end{array}$ \\
\hline $\begin{array}{l}\text { Surface package } \\
\text { Penetrator (new) }\end{array}$ & I.1, I.4, I.6 & $\begin{array}{l}\text { Descent module + } \\
\text { attitude control system } \\
\text { Scientific payload }\end{array}$ & $\begin{array}{l}20 / 65 \mathrm{~kg} \\
1 / 4 \mathrm{~kg}, 3-10 \mathrm{~W}, \mathrm{TBO}\end{array}$ & 3 & $\begin{array}{l}\text { JPL Jovian Moon Impoctor, } \\
\text { ESA JEOMA \& EMPIE studies } \\
\text { UK Penetrator consortium }\end{array}$ \\
\hline $\begin{array}{l}\text { Remote-sensing package } \\
\text { Canneras } \\
\text { (narrow-, wide-angle,stereo) } \\
\text { Doppler spectro-innager (new) } \\
\text { ENA low-energy imager } \\
\text { ENA high-energy imager } \\
\text { Y/neutron spectrometer } \\
\text { Sub-inin wave sounder (new) } \\
\text { TIR innaging spectroineter } \\
\text { UV auroral inager } \\
\text { UV imaging spectroineter } \\
\text { V/NIR imaging spectroineter } \\
\text { X-ray imaging spectrometer }\end{array}$ & 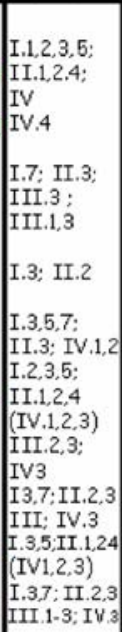 & $\begin{array}{l}400-950 \mathrm{~nm} \\
430-520 \mathrm{~nm} \\
10^{2}-10^{3} \mathrm{photons} / \mathrm{s} \\
20-5000 \mathrm{eV} \\
10 \mathrm{keV} \text { up to MeV } \\
0.1-10 \mathrm{MeV} \\
\text { 5cintillotion or Ger monium } \\
60-600 \mu \mathrm{m} \\
5-15 / 20 \mu \mathrm{m} \\
\\
90-170 \mathrm{~nm} \\
50-200 / 300 \mathrm{~nm} \\
0.5-5 \mu \mathrm{m} \\
0.17-1.5 \mathrm{~nm} \\
0.5-7.5 \mathrm{keV}\end{array}$ & 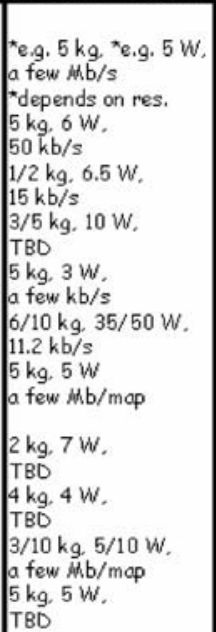 & $\begin{array}{l}3 \\
3 \\
3 \\
3 \\
3 \\
3\end{array}$ & $\begin{array}{l}\text { SIMBIO-SYS on BepiColombo } \\
\text { JunoCam on JUNO } \\
\text { Thermal, SA } \\
\text { SYMPA EB network } \\
\text { Thermal, SA } \\
\text { SERENA on BepiColombo } \\
\text { Purging after launch } \\
\text { INCA on Cossini } \\
\text { MGNS on BepiColombo } \\
\text { Thermal, Electric cleonliness } \\
\text { WIRO on Rosetta. } \\
\text { HIFI on Herschell, Thermal } \\
\text { MERTIS on BepiColombo } \\
\text { Thermal, SA } \\
\text { KuaFu STP imager (phose A) } \\
\text { Thermal, SA } \\
\text { ALICE on Rosetta. PHEBUS } \\
\text { on BepiColombo, Thermal, SA } \\
\text { SIMBIO-SYS on BepiColombo } \\
\text { JIRAM on Juno, Thermal, SA } \\
\text { MIXS on BepiColombo } \\
\text { Thermal, SA }\end{array}$ \\
\hline $\begin{array}{l}\text { Energetic particle detectors } \\
\text { INMS } \\
\text { Magnetometers } \\
\text { Plasma spectroneters } \\
\text { Radio \& Pleama Wove instrument }\end{array}$ & $\begin{array}{l}\text { (I.3): } \\
\text { II.2.4:(III) } \\
\text { I.3: } \\
\text { II.2.4: } \\
\text { I.3.7: II.3: } \\
\text { III } \\
\text { I.3.7: II.3 } \\
\text { III.3 } \\
\text { I.1,2,6.7: } \\
\text { II.1,3: III } \\
\text { I.3,7: II.3 } \\
\text { III } \\
\text { I.7: II.3: } \\
\text { III: IV.2 }\end{array}$ & $\begin{array}{l}\mathrm{m} / \Delta \mathrm{m}>100 \\
10<\mathrm{m} / \mathrm{q}<300 \\
\text { Electron/ion sensors } \\
10 \mathrm{keV}-\text { several MeV } \\
1-100 \mathrm{amu} \\
\\
\text { Static }+ \text { search coil } \\
+2-3 \mathrm{~m} \text { boom (TBD) } \\
\text { Electron/ion sensors } \\
1 \text { eV-50 keV } \\
\text { DC-40 MHz B, - } 1 \text { Mz E } \\
\text { Booms/wire antennos } \\
+ \text { Langmuir probe }\end{array}$ & 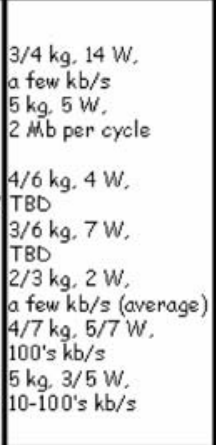 & \begin{tabular}{|c}
5 \\
3 \\
5 \\
3 \\
$3 / 5$ \\
5 \\
$3 / 5$
\end{tabular} & $\begin{array}{l}\text { Galileo/Ulysses/Cossini/Rosetta } \\
\text { Ptolemy GCMS on Philoe } \\
\text { (ACP on Huygens), generates } \\
\text { radiofrequency, Thermal, SA } \\
\text { MIMI on Cassini } \\
\text { INMS on Cossini } \\
\text { Open or close ion source } \\
\text { WAG on Cassini, Venus Express } \\
\text { Mhagnetic cleanliness, Thermal } \\
\text { ASDERA an Mars/VenusExpress } \\
\text { CAPS on Cassini } \\
\text { PWI on BepiColombo } \\
\text { E/B cleanliness }\end{array}$ \\
\hline
\end{tabular}

SC=spacecraft (3=3- $a x i s ; ~ S=s p i n n e r) ; S A=S$ in Avoidance; EB=Earth-Based; INMS S=Ion \& Neutral Mass Spectrometer; TBD=To Be Defined; res, =resolution; $B=$ Magnetic field; $E=E l e c t r i c$ field; ENA=Energetic Neutral Atom

The actual core payloads for each spacecraft can be extracted from this list once the available $\mathrm{p} / \mathrm{l}$ resources are determined. The color code used is the same as in traceability matrix. Instruments that have never flown in the jovian environment are underlined, new ones are indicated. A detailed description of these instruments is available at http://jupiter-europa.cesr.fr 
In addition, our payload complement includes specific high-priority instruments which require feasibility studies in order to demonstrate their ability to achieve the required science performance. Finally, we recommend inclusion of scenariodependent instruments in case it is demonstrated during the assessment phase that our proposed expanded options are achievable.

\subsubsection{Scientific payload mass allocation and shielding strategy}

Preliminary investigations have been carried out in order to assess the scientific payload mass allocation on each platform and for each scenario. The resulting minimum/maximum achievable payload mass allocations including shielding are detailed in Table 2. The lower values arise from the consideration of a 5\% launch mass margin at platform level and are the ones taken into account for the definition of our core payload complement. Our proposed additional complementary instruments, listed in order of priority, should be considered together with the maximum payload mass allocations. The stringent radiation environment around Jupiter forces us to consider high shielding masses for each of the instruments. Since definitive specification levels are not yet available, we chose to introduce realistic shielding coefficients in order to ensure we do not underestimate this issue. Therefore, we reduce payload mass allocations by $20 \%$ both on JPO and JMO, and by $40 \%$ on JEO in the case of scenarios 1 and 2 , and by $20 \%$ on JPO and $30 \%$ on JEO in the case of scenario 3 since we target a shorter lifetime for the latter spacecraft. At the end, these reductions enable us to consider the instrument masses given in Table 2 in order to fit our proposed core payload complement with the resulting mass budget.

\subsubsection{Mission element strategy}

The science objectives of LAPLACE will only be met by a combination of measurements from the various instruments onboard all the mission elements. In order to optimize scientific return, we have selected our list with the objective of maximizing complementarities and synergies between individual instruments, between different platforms and to each specific target. We have also made use of the specific pointing requirements of each platform (e.g. Section 4), by allocating preferably nadirpointing instruments to JEO, remote sensing instruments to JPO, and particles-andfields instruments to JMO.

Our payload mass allocations and shielding strategy result in similar payload mass allocations on the JEO for all three scenarios. Therefore, we propose at this stage to include the same core payload complement independently of the scenario considered. In the case of the JPO, our proposed core payload complement is very similar between scenarios 1 and 2 except on two counts. First, we propose to include a more reduced plasma package for scenario 1 compared to scenario 2 since the former scenario enables us to include the JMO with a full magnetospheric instrument suite. Second, we choose to allocate more mass to the cameras for scenario 1 compared to scenario 2 in order to achieve a better resolution. Whereas our core payload complement does not differ significantly between scenarios 1 and 2, this is not the case for our proposed additional instruments, notably in term of prioritization. In the case of scenario 1, our first priority is given to a high-energy neutral atom imager on the JPO since this instrument will provide the overall context of the magnetospheric in-situ measurements taken from the instruments on the JMO. In the case of scenario 
Table 2 Proposed set of science payload elements for each spacecraft and for each scenario

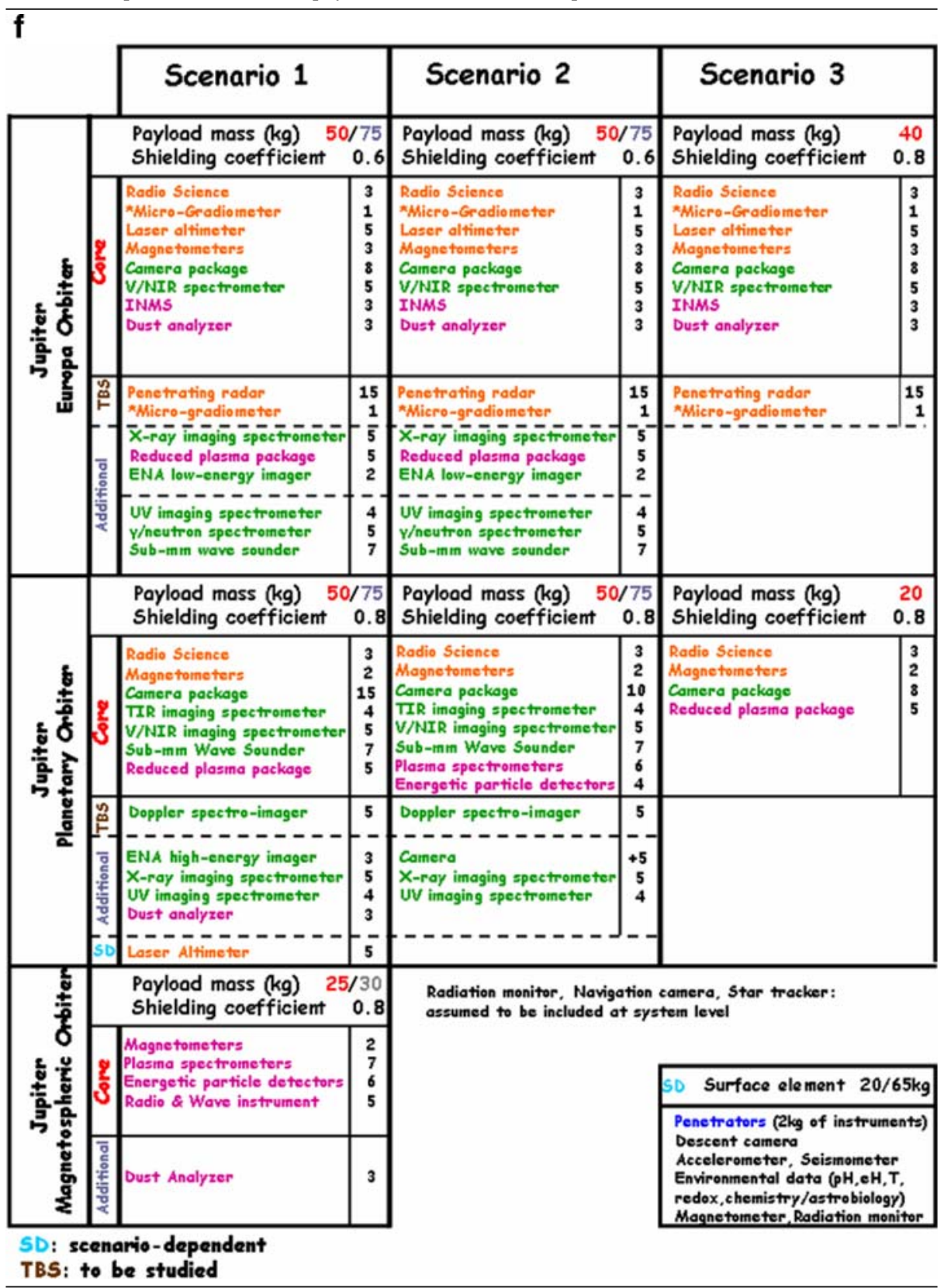

Core scientific instruments have been chosen to ensure the prime science objectives of LAPLACE are addressed, as well as fitting with the preliminary lower mass budget offered by each mission element. Additional complementary instruments are also proposed, if higher mass budgets are achievable. To be studied and scenario-dependent instruments are also included. Color codes are used to designate the three main categories of instruments 
2, our first priority is given to supplementary mass allocation for the cameras. Finally, our proposed payload complement on the JPO for scenario 3 is severely constrained by the limited achievable payload mass allocation.

One last justification for our proposed payload complement needs to be highlighted. The LAPLACE mission will enable for the first time multi-point measurements of the jovian system to be obtained.

To take advantage of this unique opportunity, we need to include similar instruments on-board several, if not all, of our mission elements, independent of the scenario considered. This concerns particularly the magnetospheric in-situ instruments. Magnetometers are essential instruments since they address both the planetary and magnetospheric objectives of the LAPLACE mission. It seems therefore logical to include such instrumentation on each platform. Charged-particle detectors covering energy range from a few eV to several $\mathrm{MeV}$ will be carefully designed to provide detailed information on composition and charge state of the jovian magnetospheric plasma. These key measurements will enable us to go beyond the limited or incomplete measurements provided by the various spacecrafts which previously investigated the jovian magnetosphere. It is particularly appropriate to include such instruments on each platform, even with reduced capabilities. Second, such an opportunity concerns also the cameras since they will enable us to observe simultaneously different or similar locations onto the surfaces of the jovian satellites, at different resolutions. Since the JPO will spend most of its orbital time at moderate but not close distances from the jovian satellites, we ensure that onboard framing cameras will be able to observe these objects with enough resolution and allocate them in consequence a larger mass than on the JEO. As a general rule, the more payload mass is available, the more is allocated to the cameras.

\subsubsection{Specific instruments to be studied during the assessment phase}

Penetrating radar A ground penetrating radar is a very attractive but challenging instrument that could provide a definitive answer concerning the presence of a subsurface ocean at Europa as well as a unique diagnosis of the structure of the ice shut. Following the promising conclusions of the dedicated ESA CDF study 'Europa Low Resource Radar', a further instrument assessment study is recommended. On scientific aspects, a full-scale sensitivity study is required to estimate the effects of the external and internal structure of Europa (ionospheric effects, radio noise, radiation environment, surface properties, temperature profiles and ice dielectric properties) that can affect significantly the radar performances. Current models should be consolidated by analyzing all related data returned by the Galileo spacecraft. On technical aspects, further studies on the design, accommodation, deployment and data commissioning of the instrument are required. The feasibility study should also analyse the possibility of using the altimetric mode of this instrument.

Micro-gradiometer A micro-gradiometer is expected to bring strong scientific added-value for the study of the internal structure of Europa by obtaining highresolution gravity field information. But its implementation on the JEO imposes strong constraints on the spacecraft design. An instrument assessment study, following the dedicated ESA study 'Gravity Sensor Technology for Future Planetary Mission', is required to demonstrate that it can meet its science requirements: the instrument has to be exactly at the centre of mass of the spacecraft, or, alternatively, 
the position of its centre of mass has to be precisely monitored. Moving parts in the spacecraft (e.g., propellant in the tanks) must be precisely monitored in order to correct the gravity gradient measurements. This instrument requires a very accurate knowledge of the attitude (angular velocities and angular accelerations, which are part of the measurements), and an in-flight calibration system/strategy. In case this instrument can not be implemented on JEO, we will rely on a conservative approach based on classical Earth-based tracking (Radio Science experiment) in order to determine the gravity field of Europa and its time variation due to tidal deformation.

Doppler spectro-imager The roadmap we are planning for jovian seismology will include a feasibility study, both to assess the feasibility of a space version of a Doppler velocity imager (with possible heritage from very similar helioseismic experiment) and to prove the capability of the space instrument to achieve the required performance for detection of the jovian oscillations. We are also considering the possibility to apply for support from the Framework Programme 7 Space ThemeStrengthening of Space Foundations Activity. The feasibility study will also include the analysis of the possibility to integrate the Doppler imager as a specific visible channel within a fully integrated spectrograph/camera package, allowing one to consider it as a possible option for scenario 2.

Penetrators For penetrators for the Europan surface the feasibility of such instrumentation requires analysis; confirming survival to very high-speed impacts and operations lifetime issues (thermal concerns due to cold conditions, insulation, batteries, RHU); communications (surface material transparency to radio, need for a trailing aerial). The ruggedness of seisometers is an issue as is heat flow measurement feasibility. An initial study of a mineralogy/astrobiology instrument should be carried out as well as miniature attitude control systems and selection and development of a de-orbit motor.

\subsection{Critical issues - the radiation environment and a highly integrated payload approach}

A mission to the Jupiter system presents important technical and engineering challenges. The limited payload mass coupled with low power levels and the harsh radiation environment dictate that the design of both the spacecraft and the accommodation of science instruments follow a new approach. A Robust, Efficient, Autonomous Payload approach (REAP) hereafter referred to as a highly integrated payload will maximize the LAPLACE mission science return in two ways: (1) by minimising overlapping resources and optimising the sharing and utilisation of available hardware within the payload; (2) the risks of premature instrument or mission failure due to radiation effects will be minimised by an adequate design of spacecraft electronics. Space electronics and in particular mass memory and processors are susceptible to a number of radiation induced effects which can lead to a variety of performance reduction and failure modes. These radiation effects are broadly separated into two categories: (a) Total Ionising Dose (TID) damage and (b) Single Event Effects (SEE). TID damage presents itself as a gradual degradation in operation and performance of electronic components and can have a considerable effect on the power production efficiency of solar panels. It is the less serious of the 
two effects and can be minimised and even reversed by various strategies such as minimal component power-on time and thermal annealing cycles. Also of concern is the SEE rate of mission critical components. A radiation induced SEE such as Single Event Latchup (SEL) could lead to a mission critical failure due to component burnout in the power or communications system for example and a SEE within a mission critical segment of the spacecraft memory could lead to software corruption with associated serious effects. It should be noted that the use of silicon-on-insulator (SOI) substrates can reduce component sensitivity to SEE's.

As an example of the radiation environment, the TID behind $8 \mathrm{~mm}$ of Al shielding accumulated from a 66 day tour of Europa is of the order of 1 Mrad. A comfortable level of margin will therefore demand hardware that is hardened to the several Mrad level. This dose is well beyond the level at which current space qualified mass memory, processors and other components are rad hardened. The full realisation of the Jupiter mission science goals and those of the Europa orbiter (JEO) in particular, will rely on a trade off between spacecraft shielding and the radiation tolerance of the electronics. Any reduction in shielding requirements can be translated into an increased instrument capacity and thus science return. The use of alternative shielding materials such as Ta coupled with a multi-layered approach with different materials can serve to both reduce mass and increase the radiation resistance over a broader range of particle energies. Previous studies have shown that solar cells are also susceptible to degradation in an intense radiation environment with the result being an appreciable decrease in output power. A $1 \mathrm{MeV}$ electron flux at Europa $\left(9.3 \mathrm{R}_{J}\right)$ of $\sim 10^{13} \mathrm{~cm}^{-2}$ day $^{-1}$ integrated over a 60 day tour will lead to a $20 \%$ reduction of the solar cell output, which will have to be taken into account during mission planning and operations.

In the REAP approach, in order to minimize the risk of instrument failure, as much of the instrument as possible should be enclosed within a shielded environment with only the front end sensor exposed to the environment. This co-location of a large portion of the instruments also provides the opportunity to share resources; centralized DC/DC conversion for common electronic component standard voltages being one example. This resource requirement reduction is complementary with the second benefit of the REAP approach which is a high level of hardware redundancy. The instruments under the REAP approach will have access to multiple processors with a single processor at a time being operational (depending on the overall instrument suite processing load). If a processor displays signs of radiation induced degradation then a replacement will be automatically assigned to service the instruments. A mechanism providing automatic switch over of the failed components without damage in the switchover operations will be central to the implementation of the REAP architecture.

Table 3 illustrates the mission benefits gained from a highly integrated payload approach: it will make it possible to include in the core payload a number of the additional complementary instruments introduced in Table 2, thereby increasing significantly the science return of the LAPLACE mission. As a first step and only for illustrative purposes, we introduce in Table 3 two categories of highly-integrated instrument package, a remote sensing one (consisting of optical and spectroscopic instruments) and a magnetospheric one (consisting of fields and particles instruments). We also propose an attempt to combine the laser altimeter receiver with a camera, for a targeted mass of $7 \mathrm{~kg}$. 
Table 3 Proposed set of science payload elements for each spacecraft and for each scenario

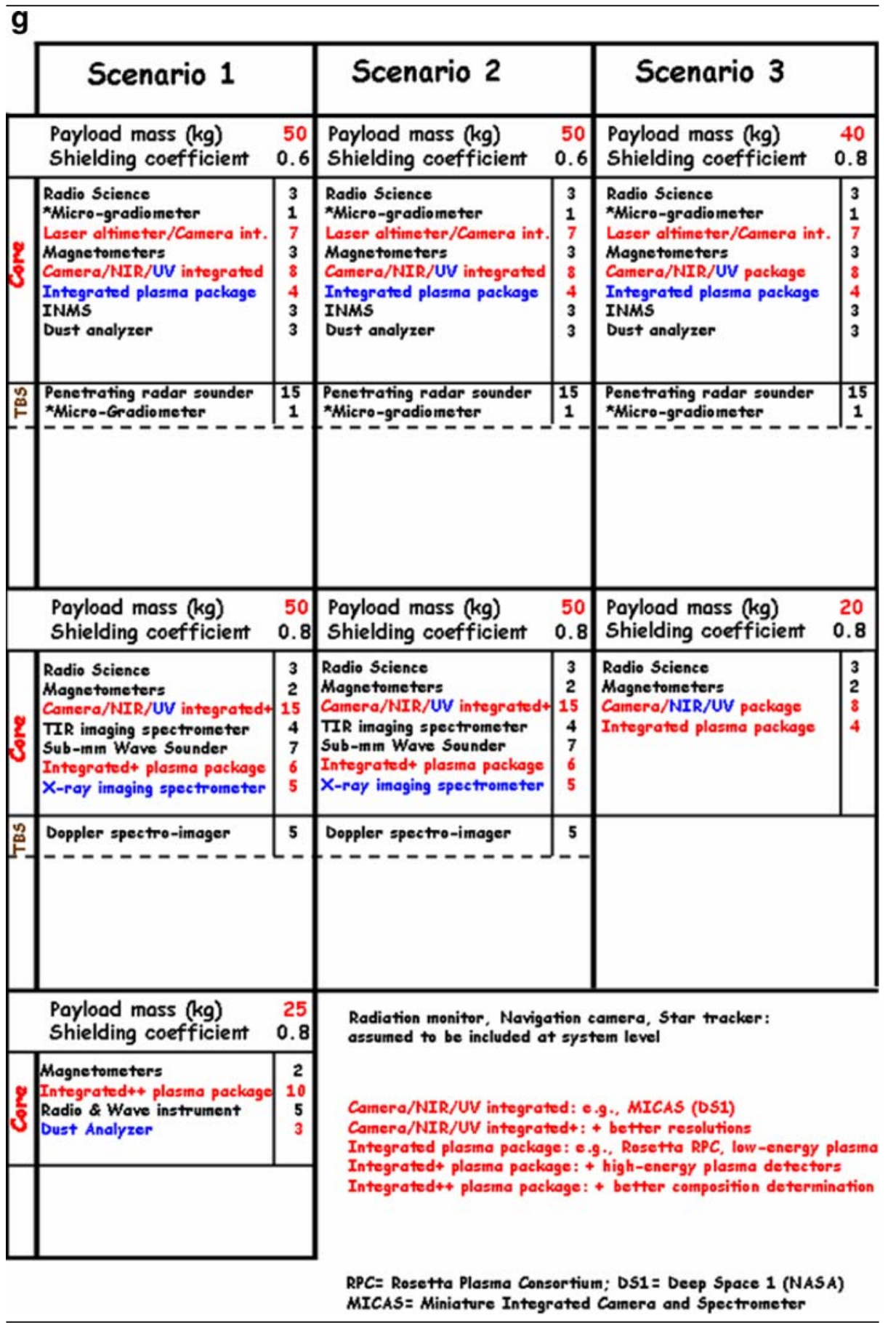

Compared to Table 2, highly-integrated instrument packages have now been introduced and make it possible to include as core payload several of the additional complementary instruments listed in Table 2, for the same payload mass budgets 
Our proposed camera and spectrometer compact packages (N.B.: each with different resolutions, hence different focal lengths) could be similar to the various approaches used in the case of the BepiColombo SIMBIO-SYS or proposed in the case of the Pluto Express HIPPS or Deep Space 1 MICAS packages. The latter attempted to combine two cameras, an ultraviolet imaging spectrometer and an infrared imaging spectrometer plus all the thermal and electronic control for $12 \mathrm{~kg}$, all its sensors sharing a single 10-cm diameter telescope.

Our proposed magnetospheric packages (N.B.: each with different performances, without including a magnetometer) could be similar to the approach developed and used in the case of the Rosetta-RPC package, which combines five sensorsion and electron sensors, an ion composition analyzer, a Langmuir probe, a mutual impedance probe together with a magnetometer-for $7.45 \mathrm{~kg}$ and $12.1 \mathrm{~W}$.

In conclusion, use of the REAP approach clearly enhances the potential science return of LAPLACE, and is highly recommended for its implementation.

\section{Summary and conclusions}

LAPLACE is a multi-platform mission aiming at an in-depth, quantitative study of the Jupiter system and its moons, with special emphasis on Europa, which focuses on three major and interrelated scientific questions.

1. What have been the conditions for the formation of the Jupiter system?

2. How does the Jupiter system work?

3. Is Europa habitable?

To address these three key questions, our observation strategy will use three orbiting platforms: a Jupiter-Europa Orbiter (JEO), and two Jupiter orbiters: a Jupiter Planetary Orbiter (JPO) and a Jupiter Magnetospheric Orbiter (JMO). Our exploration of Europa and the Jupiter System will proceed by successive phases, prepared and supported by adequate Earth-based observations using the major facilities of the time. The cruise phase will provide an opportunity for detecting the global modes of oscillation of Jupiter. After Jupiter Orbit Insertion, a multiple-year orbital tour by the three independent platforms will follow, during which in-depth, multi-spacecraft studies of Jupiter's satellites, atmosphere and magnetosphere will be performed. The "core" Europa mission will then be performed by injecting the JEO on a polar circular Europan orbit to study Europa's surface, sub-surface, ocean structure and floor, interior and exosphere, and to assess its habitability, for a period of at least a few months. The possibility of carrying a surface element will be studied during the assessment phase. After completion of the Europa mission by crashing of the JEO on the satellite's surface, the investigation of the Jupiter system will continue for at least 2 years with JPO and JMO, with the possibility of injecting the JPO on a Ganymede orbit at the end of its mission.

The measurement requirements of LAPLACE will be satisfied using three complementary categories of scientific investigations:

- measurements of Europa's geometry and its main planetary fields and to a lesser extent those of the other satellites (gravity, magnetic field, geodesy and surface topography), including their response to tidal forcing, to retrieve the 
main characteristics of Europa's ocean and determine the internal structure of the different satellites,

- multi-spectral remote sensing measurements of the surfaces, atmospheres and gas tori, and possibly sub-surfaces of the different bodies of the Jupiter system, to retrieve their composition and dynamics and improve our understanding of the vertical coupling mechanisms between the different layers,

- in situ remote sensing of the plasma, fields, energetic particles gas and dust populations of Europa's exosphere and Jupiter's magnetosphere, to quantitatively understand the mutual interactions between the different bodies of the Jupiter system and its space environment.

A mission to Europa and the Jupiter System offers a set of very stimulating technological challenges. These include high-precision navigation necessary to retrieve the internal structure of the satellites, on-board solar power and possibly nuclear energy sources, data transmission and storage capabilities, protection of on-board systems and instruments against the extremely high radiation doses produced by Jupiter's radiation belts, and the compliance with Planetary Protection standards.

The broad spectrum of scientific themes and of technological challenges, its multiplatform architecture, and the large community of interested disciplines, scientists and institutes in Europe and abroad, all point to the strong benefit that such a mission will draw from international, possibly world-wide collaboration. Potential partners for ESA (NASA, JAXA, RosCosmos and beyond) have been identified, together with corresponding collaboration scenarios.

The discovery of Jupiter's main satellites by Galileo Galilei in 1610 was made possible by the invention of the telescope-a milestone in the understanding of Cosmos by mankind. The fact that 400 years later the first observational objects of the first telescope are becoming subjects of detailed in-situ investigation by the LAPLACE mission has a huge educational potential. The mission will exploit this potential emphasizing the mutually beneficial interaction between astronomy, planetary and space science on one hand and cutting edge developments in technology on the other hand. It will associate from its early study and development phases the public and the education bodies in Europe and beyond, building a bridge across four centuries between one of the founding events of modern astronomy and one of the most challenging scientific and technological endeavors of our time: exploring distant words, to better understand our own Solar System, its origin and its destiny.

Acknowledgements The LAPLACE team expresses its sincere gratitude to CNES PASO, EADS/Astrium, Noveltis and ThalesAleniaSpace for their very useful support to the design of this proposal.

\section{Appendix: A possible Europa Surface Element: the mission proposed by Roscosmos}

The existence of an aqueous ocean, determination and mapping of ice thickness, interactions between ice crust and liquid, liquid and silicate mantle, chemical characterization of ice surface and correlation with surface imaging (inorganics, organics, isotopic fractionation) and the measurements of volatiles $(\mathrm{CH} 4, \mathrm{NH} 3, \mathrm{CO} 2$, etc) represent important scientific objectives of the LAPLACE mission. Many of these 
objectives may be achieved by experiments performed directly at the surface of Europa. Moreover, if Europa is (or was) habitable, a logical exploration step should be the search for any signature of life that the environment is able to sustain. Use of a Surface Element (SE) would be particularly relevant in this perspective.

This is why, as a complement to the LAPLACE mission, Roscosmos and the Russian Academy of Sciences consider an independent Russian segment of the mission with the objective of delivering a Surface Element to Europa. This segment will include a transfer vehicle separately launched by a Soyuz-class launcher allowing a descent module for Europa with a primary scientific goal of in situ studies at the surface. It will rely on the LAPLACE orbiters to relay the data from the Russian SE. A broad international involvement in the science payload and exploration tools of the descent module is envisaged.

In situ studies on the surface and shallow subsurface of Europa will allow a detailed characterization of the surface material, and will provide insights into the origin of the icy satellites, via analysis of noble gases isotopic record. Therefore the emphasis of the SE payload is made at a hi-end GC/MS of evolved gases capable to characterize noble gases isotopic composition. A number of additional instruments will contribute to determination of elemental/chemical composition of the surface material. Assuming that the microbe life has aroused in ocean, the remains of microbes and its fragments and complex organic compounds could be also present in surface ice. Such signatures could be identified by measuring the elements of biological significance $(\mathrm{C}, \mathrm{O}, \mathrm{N}, \mathrm{H}, \mathrm{P}, \mathrm{S}, \mathrm{Na}, \mathrm{K}, \mathrm{Ca})$ and detailed chemical analysis.

The scientific payload will include a subsystem for preparation of the surface sample. Depending on the available resources, alternatively a combination of grinder with robotic arm, or drill/thermal penetration system is envisaged. A sample preparation line will feed GC/MS of evolved gases with a possibility of isotope/ noble gases analysis. Prior to this destructive analysis the samples may be examined using visible and IR microscopy, Raman spectroscopy, and UV or X-ray fluorescence method. The same sample preparation line will serve for a comprehensive instru-

Fig. 11 Baseline Europa landing platform from Phobos heritage

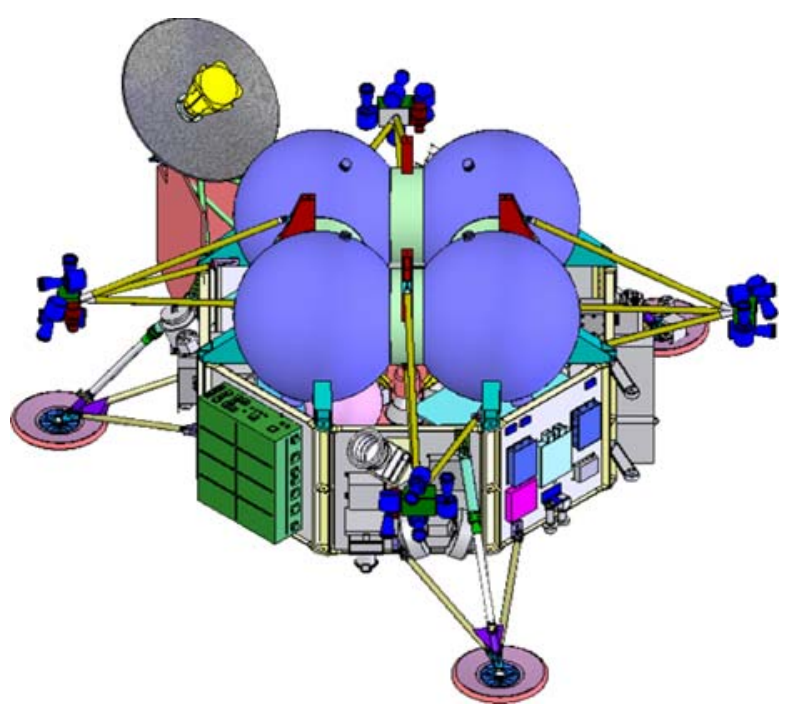


mentation for chemical/biological characterization of the ice, possibly including immuno-assemblies, liquid chromatography, and tests for metabolic activity. Some active experiments, such as APXS, Laser TOF mass-analyzer, and Laser ablation spectrometer may be performed directly on the surface. Investigations of Europa's internal structure by means of a dedicated seismology experiment are a must for the lander. A seismometer will detect without ambiguity the position of the ocean interface, providing direct evidence for a sub-surface ocean, and may constrain the silicate mantle depth. Finally, the science instrument set on the lander will include a panoramic/context camera, and a set of sensors to characterize the environment (temperature, radiation, etc.)

The access to subsurface of Europa, aiming at studying pristine material not modified by radiation, even at a depth of a few $\mathrm{cm}$ is a challenging task because of the high stiffness of ice at low temperature. Different descent vehicles, including small stations, a larger landing platform, and penetrators were considered. The present scenario of the Russian segment of the Laplace mission, which will enter Phase A in 2008 in Russia, is as follows:

- Soyuz launch from Baikonur;

- Earth-Jupiter cruise phase, approach and Europa orbit insertion, similarly to LAPLACE;

- Europa orbiting phase (to be coordinated with LAPLACE scenario) and remote sensing studies;

- A small station equipped with an abrasion tool or other sampling means performs soft landing in an area selected for its potential exobiological and geological interests.

For a landing to Europa, methods and algorithms of soft landing to the Moon employed in earlier Soviet lunar missions are fully applicable. The mass budget for the landing platform, estimated on the basis of lunar landing platforms and subsystems recently developed for Phobos Sample Return mission, is $400 \mathrm{~kg}$ with a possibility to accommodate more than $20 \mathrm{~kg}$ of scientific instruments (Fig. 11).

Open Access This article is distributed under the terms of the Creative Commons Attribution Noncommercial License which permits any noncommercial use, distribution, and reproduction in any medium, provided the original author(s) and source are credited.

\section{References}

1. Achilleos, N., Miller, S., Tennyson, J., Aylward, A.D., Mueller-Wodarg, I., Rees, D.: JIM: a timedependent, three-dimensional model of Jupiter's thermosphere and ionosphere. J. Geophys. Res. 103(E9), 20089-20112 (1998)

2. Alibert, Y., Mousis, O., Mordasini, C., Benz, W.: New Jupiter and Saturn formation models meet observations. Astrophys. J. 626, L57-L60 (2005)

3. Alibert, Y., Mousis, O., Benz, W.: Modeling the Jovian subnebula. I. Thermodynamic conditions and migration of proto-satellites. Astron. Astrophys. 439, 1205-1213 (2005)

4. Andre, N., Ferriere, K.M.: Low-frequency waves and instabilities in stratified, gyrotropic, multicomponent plasmas: theory and application to plasma transport in the Io torus. J. Geophys. Res. 109, A12225 (2004)

5. Atreya, S.K., Wong, M.H., Owen, T.C., Mahaffy, P.R., Niemann, H.B., de Pater, I., Encrenaz, Th., Drossart, P.: Comparison of the atmospheres of Jupiter and Saturn: deep atmospheric composition, cloud structure, vertical mixing, and origin. Planet Space Sci. 47, 1243 (1999) 
6. Bagenal, F., Dowling, T.E., McKinnon, W.B. (eds.): Jupiter: The Planet, Satellites and Magnetosphere. Cambridge University Press, Cambridge (2004)

7. Boss, A.P.: Collapse and fragmentation of molecular cloud cores 2: collapse induced by stellar shock waves. Astrophys. J. 439, 224-236 (1995)

8. Boss, A.P.: Giant planet formation by gravitational instability. Science 276, 1836 (1997)

9. Bougher, S.W., Waite, J.H., Majeed, T., Gladstone, G.R.: Jupiter Thermospheric General Circulation Model (JTGCM): global structure and dynamics driven by auroral and Joule heating. J. Geophys. Res. 110(E4) (2005)

10. Canup, R.M., Ward, W.R.: Formation of the Galilean satellites: conditions of accretion. Astron. J. 124, 3404-3423 (2002)

11. Canup, R.M., Ward, W.R.: A common mass scaling for satellite systems of gaseous planets. Nature 441, 834-839 (2006)

12. Carlson, R.W., et al.: Distribution of hydrate on Europa: further evidence for sulfuric acid hydrate. Icarus 177(2), 461-471 (2005), Oct 2005

13. Carr, M.H., et al.: Evidence for a subsurface ocean on Europa. Nature 391(6665), 363-365 (1998)

14. Castillo, J., Mocquet, A., Sotin, C.: Detecting a deep ocean within Europa by means of geodetic measurements. C. Rend. Acad. Sci. 330(9), 659-666(8) (2000)

15. Chust, T., Roux, A., Kurth, W.S., Gurnett, D.A., Kivelson, M.G., Khurana, K.K.: Are Io's Alfven wings filamented? Galileo observations. Planetary Space Sci. 53, 395-412 (2005)

16. Chyba, C., Ostro, S., Edwards, B.: Radar detectability of a subsurface ocean on Europa. Icarus. 134, 292-302 (1998)

17. Chyba, C.F.: Energy for microbial life on Europa-a radiation-driven ecosystem on Jupiter's moon is not beyond the bounds of possibility. Nature 403(6768), 381-382 (2000)

18. Chyba, C.F., Phillips, C.B.: Possible ecosystems and the search for life on Europa. Proc. Natl. Acad. Sci. U. S. A. 98(3), 801-804 (2001)

19. Cooper, J.F., et al.: Energetic ion and electron irradiation of the icy Galilean satellites. Icarus 149(1), 133-159 (2001)

20. Des Marais, D.J., Walter, M.R.: Astrobiology: exploring the origins, evolution, and distribution of life in the Universe. Ann. Rev. Ecol. Syst. 30, 397-420 (1999)

21. Des Marais, D.J., et al.: The NASA astrobiology roadmap. Astrobiol. 3-2, 219-235 (2003)

22. Dessler, A.J.: Preface, Physics of the Jovian Magnetosphere. Cambridge University Press, New York (1983)

23. Divine, N., Garrett, H.B.: Charged particles distributions in Jupiter's magnetosphere. J. Geophys. Res. 88, 6889 (1983)

24. Drossart, P., et al.: Detection of $\mathrm{H}_{3}^{+}$on Jupiter. Nature 340, 539 (1989)

25. Flasar, M., CIRS Team: Temperature, winds, and composition in the Saturnian System. Science 307, 1247 (2005)

26. Geissler, P.E.: Volcanic activity on Io during the Galileo Era. Annu. Rev. Earth Planet. Sci. 31, 175-211 (2003)

27. Goldreich, P., Lynden-Bell, D.: Io: a Jovian unipolar inductor. Astrophys. J. 159, 59-78 (1969)

28. Greeley, R., Chyba, C.F., Head III, J.W., McCord, T.B., McKinnon, W.B., Pappalardo, R.T., Figueredo, P.H.: Geology of Europa. In: Bagenal, F., Dowling, T.E., McKinnon, W.B. (eds.) Jupiter: The Planet, Satellites and Magnetosphere, pp. 329-362. Cambridge University Press, Cambridge (2004)

29. Greenberg, R.: Orbital evolution of the Galilean satellites. In: Morrison, D. (ed.) Satellites of Jupiter, pp. 65-92. Univ. Arizona Press, Tucson (1982)

30. Greenberg, R., et al.: Habitability of Europa's crust: the role of tidal-tectonic processes. J. Geophys. Res. Planets. 105(E7), 17551-17562 (2000)

31. Greenberg, R., Geissler, P.: Europa's dynamic icy crust. Meteorit. Planet. Sci. 37, 1685-1710 (2003)

32. Guillot, T., Stevenson, D.J., Hubbard, W.B., Saumon, D.: In: Bagenal, F., Dowling, T.E, McKinnon, W.B. (eds.) Jupiter. Cambridge Univ. Press, New York (2004)

33. Gurnett, D.A., Kurth, W.S., Roux, A., Bolton, S.J., Kennel, C.F.: Galileo plasma wave observations in the Io plasma torus and near Io. Science 274, 391-392 (1996)

34. Hill, T.W.: Inertial limit on corotation. J. Geophys. Res. 84, 6554 (1979)

35. Jakosky, B.M., Shock, E.L.: The biological potential of Mars, the early Earth and Europa. J. Geophys. Res. 103, 19359-19364 (1998)

36. Johnson, R.E., Carlson, R.W., Cooper, J.F., Paranicas, C., Moore, M.H., Wong, M.C.: Radiation effects on the surfaces of the Galilean Satellites. In: Bagenal, F., Dowling, T.E., McKinnon, W.B. 
(eds.) Jupiter: The Planet, Satellites and Magnetosphere, pp. 485-512. Cambridge University Press, New York (2004)

37. Kargel, J.S., et al.: Europa's crust and ocean: origin, composition, and the prospects for life. Icarus. 148(1), 226-265 (2000)

38. Kennel, C.F., Coroniti, F.: Is Jupiter's magnetosphere like a pulsar's or Earth's. Magnetosphere of the Earth and Jupiter. Space Sci. Rev. 17, 857-883 (1975)

39. Khurana, K.K.: Euler potential models of Jupiter's magnetospheric field. J. Geophys. Res. 102, 11295 (1997)

40. Kivelson, M.G., Khurana, K.K., Walker, R.J., Warnecke, J., Russell, C.T., Linker, J.A., Southwood, D.J., Polanskey, C.: Io's interactions with the plasma torus: Galileo magnetometer report. Science. 274, 396-398 (1996)

41. Kivelson, M.G., Khurana, K.K., Russell, C.T., Walker, R.J., Warnecke, J., Coroniti, F.V., Polanskey, C., Southwood, D.J., Schubert, G.: Discovery of Ganymede's magnetic field by the Galileo spacecraft. Nature 384, 537-541 (1996)

42. Kivelson, M.G., Khurana, K.K., Russell, C.T., Walker, R.J.: Intermittent short-duration magnetic field anomalies in the Io torus: evidence for plasma interchange? Geophys. Res. Lett. 24, 2127 (1997)

43. Kivelson, M.G., et al.: Europa and Callisto: induced or intrinsic fields in a periodically varying plasma environment. J. Geophys. Res. Space Phys. 104(A3), 4609-4625 (1999)

44. Kivelson, M.G., Khurana, K.K., Russell, C.T., et al.: Galileo magnetometer measurements: a stronger case for a subsurface ocean at Europa. Science 289(5483), 1340-1343 (2000)

45. Kivelson, M.G., et al.: The permanent and inductive magnetic moments of Ganymede. Icarus 157(2), 507-522 (2002)

46. Kivelson, M.G., Bagenal, F., Kurth, W.S., Neubauer, F.M., Paranicas, C., Saur, J.: Magnetospheric interactions with satellites. In: Bagenal, F., Dowling, T.E., McKinnon, W.B. (eds.) Jupiter: The Planet, Satellites and Magnetosphere, pp. 513-536. Cambridge University Press, New York (2004)

47. Krimigis, S.M., Carbary, J.F., Keath, E.P., Bostrom, C.O., Axford, W.I., Gloeckler, G., Lanzerotti, L.J., Armstrong, T.P.: Characteristics of hot plasma in the Jovian magnetosphereresults from the Voyager spacecraft. J. Geophys. Res. 86, 8227 (1981)

48. Louarn, P.: A study of the Jovian "energetic magnetospheric events" observed by Galileo: role in the radial plasma transport. J. Geophys. Res. 105(A6), 13073-13088 (2000)

49. Lowell, R.P., DuBose, M.: Hydrothermal systems on Europa. Geophys. Res. Lett. 32(5), L05202 (2005)

50. Magni, G., Coradini, A.: Formation of Jupiter by nucleated instability. Planet. Space Sci. 52, 343-360 (2004)

51. Marion, G.M., et al.: The search for life on Europa: limiting environmental factors, potential habitats, and Earth analogues. Astrobiol. 3(4), 785-811 (2003)

52. Mayer, L., Quinn, T., Wadsley, J., Stadel, J.: Formation of giant planets by fragmentation of protoplanetary disks. Science 298, 1756-1759 (2002)

53. McCord, T.B., et al.: Discussion of the 1.04-mu m water ice absorption band in the Europa NIMS spectra and a new NIMS calibration. J. Geophys. Res. Planets 104(E11), 27157-27162 (1999)

54. McCord, T.B., et al.: Hydrated salt minerals on Ganymede's surface: evidence of an ocean below. Science 292(5521), 1523-1525 (2001)

55. McEwen, A.S., Keszthelyi, L.P., Lopes, R., Schenk, P.M., Spencer, J.R.: The lithosphere and surface of Io. In: Bagenal, F., Dowling, T.E., McKinnon, W.B. (eds.) Jupiter: The Planet, Satellites and Magnetosphere, pp. 307-328. Cambridge University Press, New York (2004)

56. Miller, S., Aylward, A., Millward, G.: Giant planet ionospheres and thermospheres: the importance of ion-neutral coupling. Space Sci. Rev. 116(1-2), 319-343 (2005)

57. Moore, J.M., 11 colleagues: Callisto. In: Bagenal, F., Dowling, T.E., McKinnon, W.B. (eds.) Jupiter: The Planet, Satellites and Magnetosphere, pp. 397-426. Cambridge University Press, New York (2004)

58. Moore, W.B., Schubert, G.: The tidal response of Ganymede and Callisto with and without liquid water oceans. Icarus 166(1), 223-226 (2003)

59. Moore, W.B., Schubert, G.: The tidal response of Europa. Icarus 147, 317-319 (2000)

60. Morbidelli, A., Levison, H.F., Tsiganis, K., Gomes, R.: Chaotic capture of Jupiter's Trojan asteroids in the early Solar System. Nature. 435(7041), 462-465 (2005)

61. Moreno, R., Marten, A., Matthews, H.E., Biraud, Y.: Long-term evolution of CO, CS and HCN in Jupiter after the impacts of comet SL9 Shoemaker Levy SL9. Planet. Space Sci. 51(9-10), 591-611 (2003) 
62. Mousis, O., Gautier, D.: Constraints on the presence of volatiles in Ganymede and Callisto from an evolutionary turbulent model of the Jovian subnebula. Planet. Space Sci. 52, 361-370 (2004)

63. Mousis, O., Alibert, Y.: Modeling the Jovian subnebula. II. Composition of regular satellite ices. Astron. Astrophys. 448, 771-778 (2006)

64. Neubauer, F.M.: The interaction of the Galilean satellites with the Jovian magnetosphere. J. Geophys. Res. 103, 19843-19866 (1998)

65. Pappalardo, R.T., et al.: Does Europa have a subsurface ocean? Evaluation of the geological evidence. J. Geophys. Res. Planets. 104(E10), 24015-24055 (1999)

66. Pappalardo, R.T., Collins, G.C., Head III, J.W., Helfenstein, P., McCord, T.B., Moore, J.M., Prockter, L.M., Schenk, P.M., Spencer, J.R.: Geology of Ganymede. In: Bagenal, F., Dowling, T.E., McKinnon, W.B. (eds.) Jupiter: The Planet, Satellites and Magnetosphere, pp. 363-396. Cambridge University Press, New York (2004)

67. Paranicas, C., et al.: The ion environment near Europa and its role in surface energetic. Geophys. Res. Lett. 29(5), 1074 (2002)

68. Paranicas, C., et al.: Europa's near-surface radiation environment. Geophys. Res. Lett. 34(15), L15103 (2007)

69. Peale, S.J.: Orbital resonances, unusual configurations and exotic rotation states among planetary satellites. In: Burns, J.A., Matthews, M.S. (eds.) Satellites, pp. 159-223. Univ. Arizona Press, Tucson (1986)

70. Pollack, J.B., Hubickyj, O., Bodenheimer, P., Lissauer, J.J., Podolak, M., Greenzweig, Y.: Formation of the giant planets by concurrent accretion of solids and gas. Icarus 124, 62-85 (1996)

71. Pontius, D.H., Hill, T.W.: Rotation driven plasma transport: the coupling of macroscopic motion and microdiffusion. J. Geophys. Res. 94, 15041 (1989)

72. Prangé, R., Rego, D., Southwood, D., Zarka, P., Miller, S., Ip, W.: Rapid energy dissipation and variability of the Io Jupiter electrodynamic circuit. Nature 379, 323 (1996)

73. Read, P.L., Gierasch, P.J., Conrath, B.J., Simon-Miller, A., Fouchet, T., Yamazaki, Y.H.: Mapping potential-vorticity dynamics on Jupiter. I: zonal-mean circulation from Cassini and Voyager 1 data Quarterly. J. R. Meteorolog. Soc. 132, 1577-1603 (2006)

74. Reynolds, R.T., Squyers, S.W., Colburn, D.S., McKay, C.P.: On the habitability of Europa. Icarus 56, 246-254 (1983)

75. Ruiz, J.: The stability against freezing of an internal liquid-water ocean in Callisto. Nature 412(6845), 409-411 (2001)

76. Santos-Costa, D., Bourdarie, S.: Modeling the inner Jovian electron radiation belt including nonequatorial particles. Planet. Space Sci. 49, 303 (2001)

77. Schmider, F.-X., Fossat, E., Mosser, B.: Possible detection of Jovian global oscillations. Astron. Astrophys. 248, 248-281 (1991)

78. Schubert, G., Anderson, J.D., Spohn, T., McKinnon, W.B.: Interior composition, structure and dynamics of the Galilean Satellites. In: Bagenal, F., Dowling, T.E., McKinnon, W.B. (eds.) Jupiter: The Planet, Satellites and Magnetosphere, pp. 281-306. Cambridge University Press, New York (2004)

79. Shock, E.L., Holland, M.E.: Quantitative habitability. Astrobiol. 7-6, 839-851 (2007)

80. Sotin, C., Prieur, D.: Jupiter's moon Europa: geology and habitability. In: Horneck, G., Rettberg, P. (eds.) Complete Course in Astrobiology, Chapter 10. Wiley, New York (2007)

81. Spohn, T., Schubert, G.: Oceans in the icy Galilean satellites of Jupiter? Icarus 161, 456-467 (2003)

82. Tobie, G., Mocquet, A., Sotin, C.: Tidal dissipation within large icy satellites: applications to Europa and Titan. Icarus 177, 534-549 (2005)

83. Tobie, G., Choblet, G., Sotin, C.: Tidally heated convection: constraints on Europa's ice shell thickness. J. Geophys. Res. 108(E11), 10.1-10.15 (2003)

84. Van Hoolst, T., Rambaux, N., Karatekin, O.: The Hydrostatic Shape of Europa and Implications for the Satellite's Libration. AGU, Fall Meeting, Abstract \#P53B-1235 (2007)

85. Yoder, C.F.: How tidal heating in Io drives the Galilean orbital resonance locks. Nature 279, 767-770 (1979)

86. Zolotov, M.Y., Shock, E.L.: Energy for biologic sulfate reduction in a hydrothermally formed ocean on Europa. J. Geophys. Res. Planets. 108(E4), 5022 (2003)

87. Zolotov, M.Y., Shock, E.L.: A model for low-temperature biogeochemistry of sulfur, carbon, and iron on Europa. J. Geophys. Res. Planets 109(E6), E06003 (2004) 